\title{
A Wide-Ranging Review on Diabetic Retinotherapy
}

\author{
Toufique Ahmed Soomro ${ }^{1}$, Ahsin Murtaza Bughio ${ }^{2, *}$, Shahid Hussain Siyal ${ }^{3}$, Ali Anwer Panhwar ${ }^{1}$, Nas- \\ reen Nizamani ${ }^{1}$
}

${ }^{1}$ Electronics Engineering Department, QUEST, Larkana, Pakistan.

${ }^{2}$ Electronics Engineering Department, QUEST, Nawabshah, Pakistan

${ }^{3}$ Energy and Environment Department, QUEST, Nawabshah, Pakistan

${ }^{*}$ Corresponding author: ahsan.murtaza@quest.edu.pk

\section{Abstract}

\begin{abstract}
Diabetic Retinopathy (DR) is one of the major eye diseases that causes damage to retina of the human eye ball due to the rupture of tiny blood vessels. DR is identified by the ophthalmologists on the basis of various specifications i.e., textures, blood vessels and pathologies. The ophthalmologists are recently considering software for eye diseases detection based on image processing designed by the computing techniques and bio-medical images. In the analysis of medical imaging, traditional techniques of image processing and computer vision have played an important role in the field of ophthalmology. From the past two decades, there is a tremendous advancement in the development of computerized system for DR detection. This paper comprises the five parts of analysis on image based retinal detection DR, named as review of low varying contrast techniques of the retinal fundus Images (RFI), review of noise effect in the fundus images, review of pathology detection method from the retinal fundus images, review of blood vessels extraction from the RFI, and review of automatic algorithm for the DR detection. This paper presents a comprehensive detail to each problem in the retinal images. The procedures that are currently utilized to analyze the contrast issue and noise issues are discussed in detail. The paper also explains the techniques used for segmentation. In the end, the recent automated detection system of related eye diseases or DR is described.
\end{abstract}

Keywords-Retinal Images, enhancement of retinal images, normalization of retinal images, de-ionization of retinal images, automatic detection of DR, retinal images Segmentation

\section{Introduction}

$\mathrm{D}$ IABETIC Retinopathy, usually referred to as DR, is a disease linked to eyes and is impediment to Diabetes Mellitus and major reason to retinal vasculature mutilation that causes loss of sight. This silent ailment kicks in once the problems of vision loss start. The process of treating DR is complicated as it starts progressing [1]. Increase in diabetic life expectancy has risen the frequency of diabetic retinopathy. Diabetes is a major issue in the world of health-care [2]. It is predicted by World Health Organization that over next 2.5 decades, the number of diabetic patients is expected to rise from 130 million to 350 million [2].

According to Access Economics report of the Australian government in 2004, $1.6 \%$ of the people aged 40 and above suffer from DR. According to the eye

ISSN: 2523-0379 (Online), ISSN: 1605-8607 (Print)

DOI: 10.52584/QRJ.1802.25

This is an open access article published by Quaid-e-Awam University of Engineering Science \& Technology, Nawabshah, Pakistan under CC BY 4.0 International License. study of the Blue Mountains, Wales, Australia, it is indicated that one in three people with diabetics have DR. In Australia, the irreversible blindness among adults increases day by day. It is in top five of the disease list. Recently, a study reported that one in 20 diabetics patients have diabetic macular oedema or last stage of DR [3].

Diabetic Retinopathy is divided in to two categories named as Proliferative Diabetic Retinopathy (PDR) and Non-Proliferative Diabetic Retinopathy (NPDR). The classification of DR is considered by the occurrence of pathologies and rental vessels variations. NPDR contains three stages such as Mild, Moderate and Severe. One of the significant procedures is Eye screening for observing DR progress and giving essential treatment to patients for prevention of further development or deterrence to loss of vision [4].

The retinal vasculature network gives significant observation of DR progress. Doctors can observe DR development due to the retinal blood vessels' alteration 
or loss around the macula of the Foveal Avascular Zone (FAZ) of the RFI is detectable. Macula is an area of FAZ in fundus images that contains no blood vessels. The enlargement of the size of FAZ causes a blood vessel in macula area, and it progresses the disease severity level [5][6].

The Fundus Fluoresceine Angiography (FFA) represents the retinal photography. The FFA gives following three key features.

1) The way dye passes through the blood vessels to enter the retina and choroid and its circulation when it finally reaches there.

2) Fine description of retinal pigment and flow that may be invisible are observed.

3) In the resulting color fundus picture (CFI), the retinal vessel network is transparent and its functional integrity is assessed [4]

This review contains some main areas of retinal images analysis.

1) Review of low, varying contrast techniques of RFI

2) Review of noise effect in the fundus images

3) Review of the Blood Vessels extraction from RFI

4) Review of pathology detection method from the RFI

5) Review of automatic algorithm for detection of DR

The paper is structured as follows. Section 2 explains the modalities of medical images. Section 3 explains the research issues in RFI. Section 4 gives the description of biological structure of normal retina. Section 5 provides an overview of pathologies in fundus images. Severity scale of Diabetic Retinopathy is explained in section 6 . Section 7 is based on the review of computerised diagnosis for DR detection methods. Section 8 contains the review of RFI enhancement methods. The detailed review of noise effect in the fundus images is elaborated in section 9. Section 10 elaborates the retinal blood vessel segmentation. The automatic detection of Diabetic Retinopathy's review is elaborated in section 11. Section 12 contains concluding remarks.

\section{Modalities of Medical Images}

The requirement of imaging for medical diagnosis is of paramount importance. Medical imaging can be exploited to study and analyze the disease progress. Furthermore, it can also be used for the progress of medical treatment. Basically, human organs are observed with the help different modalities. Non-invasive (no surgical intervention) and invasive (surgical intervention) are the two types of medical imaging [7].

\section{1 lonizing Radiation (IR) Based Medical Imag- ing}

Ionizing radiations are dangerous to the living tissues by hurting the individual cells at the atomic level. The ionizing radiation imaging modalities are shown in Figure 1 [8].

\subsection{Non-lonizing Radiation (NIR) Based Medical Imaging Modalities}

Radio frequency ( $\mathrm{RF}$ ) waves are used for non-ionizing modalities. The $\mathrm{RF}$ waves come with the advantage of low frequency. They also have thermal as well as photochemical issues. Figure 2 shows the NIR modalities. In addition, some technical limitations can be linked with these imaging modalities are improper acquisition processes which includes poor focus and uneven radiance producing noisy image.

\section{Research Issues of RFI}

Low contrast and noisy images are the usual problems linked with the fundus images. Both these issues are raised due to imperfections in the image acquisition of the human organ. The uneven retina surface is one of the main reasons for varying contrast problems. The biological nature of the retina results in the different amounts of light being reflected that lead to a low and varied contrast image.

In Figure 3, we can see the spots which darkens the images of blood vessels leading to improper analysis. Similarly, for Green Band (GB) fundus images, desired blood vessels visibility cannot be achieved. In Figure 3, the oval shaped pigmented region near retina's centre is shown, and different regions are selected within the FAZ of the Green band of RFI. Variabiliry in the contrast is seen in macula section of the Green band fundus techniques of images are required to handle these issues.

\subsection{Low contrast in RFI}

$$
C=\frac{(\operatorname{Imax}-\operatorname{Imin})}{(\operatorname{Imax}+\operatorname{Imin})}
$$

Where Imax is the largest whereas Imin is the smallest luminous intensity values of an image. Weber's law applicable to different modalities is defined as "the contrast is the difference of two intensities divided by the initial stimulus." It is mathematically represented as,

$$
C=\frac{\Delta I}{I}
$$

where $\Delta I$ and $I$ are the luminance of the objects. An image is basically the intensity values and it comprises 


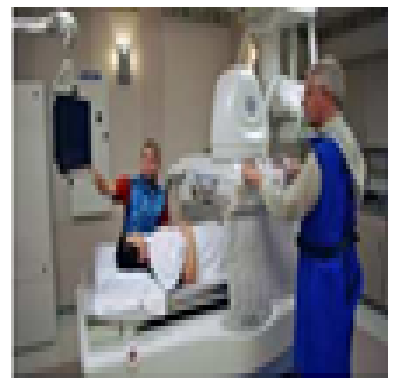

(a)

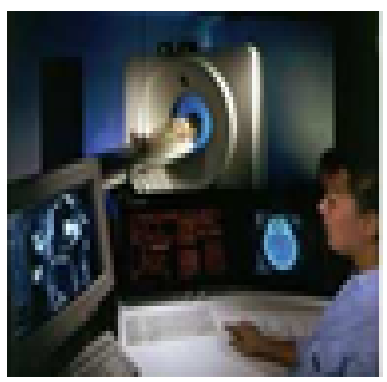

(b)

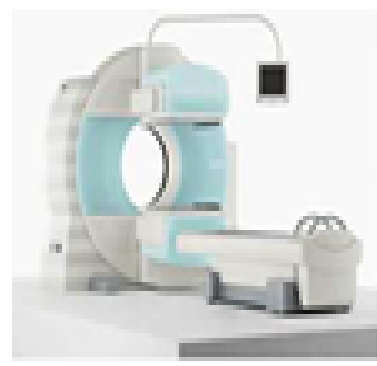

(c)

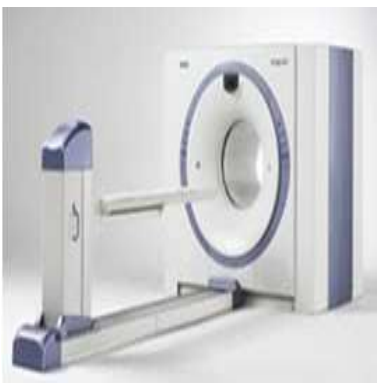

(d)

Fig. 1: (a) Fluoroscopy [9], (b) computed tomography [10], (c) Single Photon Emission Computed Tomography (SPECT) [11], (d) Positron Emission Tomography (PET) [12]

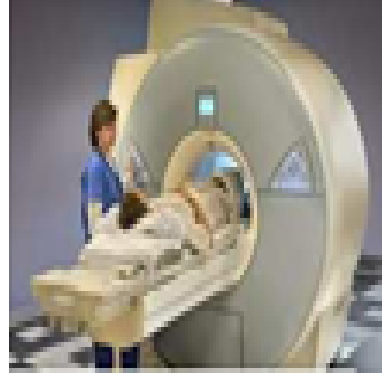

(a)

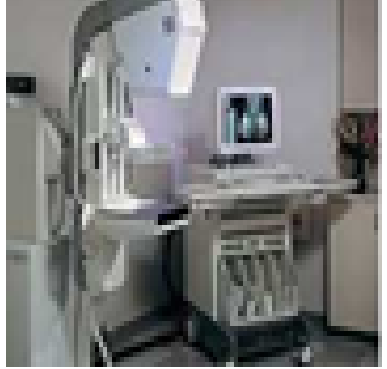

(b)

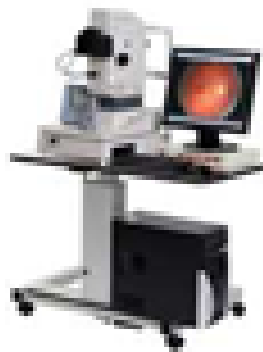

(c)

Fig. 2: (a) Magnetic Resonance Imaging (MRI) [13], (b) mammography machine [13], (c) fundus camera [14]
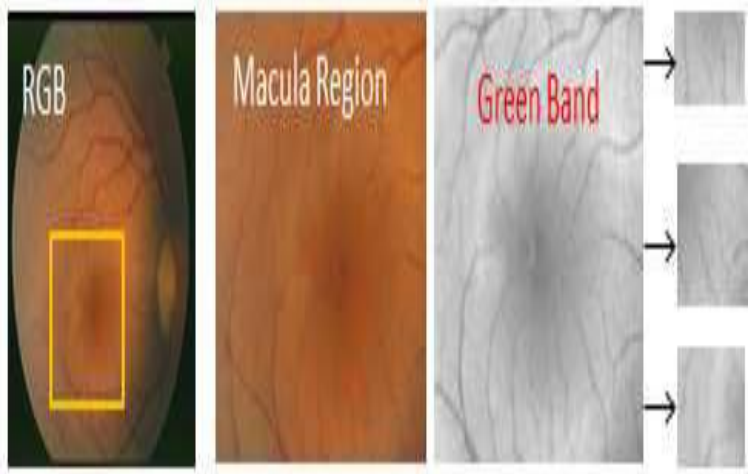

Fig. 3: Illustration of noise issue (fundus images)

definite range, and might appear in low contrast. The primary purpose of increasing the image pixels values of low contrast for clear visualisation that vessels can be achieved by doing image enhancement. Due to varying contrast issue, it is difficult to analyse accurately the retinal vasculature network [16].

\subsection{Contrast Variability Problems in RFIs}

The varying contrast in RFIs occurs because of the environmental condition of lighting and flash of the fundus camera. Uneven retina surfaces results in differing illumination [17]. Inter-varying and Intra-varying

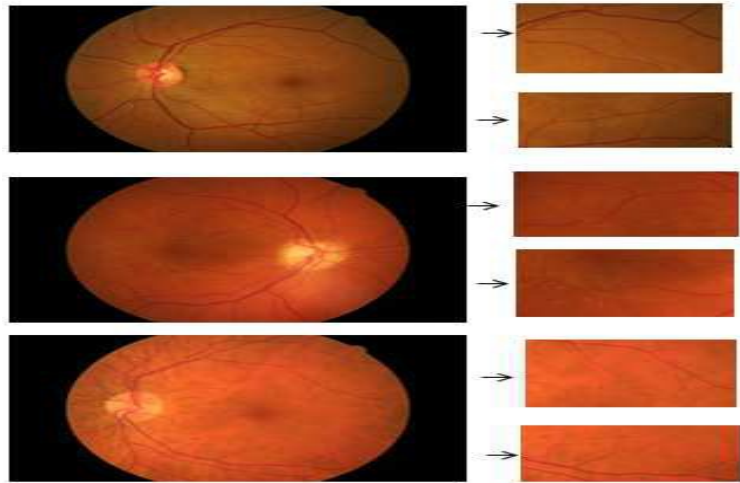

Fig. 4: Example of varied contrast in fundus Images

contrast are the types of contrast variations. RFIs of different eyes have different contrasts; this is known as inter-varying contrast and is presented in Figure 4, which shows the two types. The Fundus image macula is shown and these images are example of contrast variations showing dark and bright photos of retina.

\subsection{Noise in RFIs}

Noise can be defined as the unwanted information degrading the image quality. There are three fundamental noise models named as multiplicative, additive 
and additive plus multiplicative models. The multiplicative noise is reliant on the parameters of image; while, additive noise is naturally mechanized and can be easily exhibited [18]. The RFI comprises of the noise in retinal blood vessels images, hence, difficult to be analysed for fundus as well as GB images as shown in Figure 5.

\section{Biological Structure of Normal Retina}

Analysis of fundus image helps analyzing various eye related diseases. The two methods of capturing the fundus image are FFA (invasive) method and noninvasive techniques. In FFA, dye made of fluorescein is injected into minuscule blood vessels of eye to boost up retinal vasculature contrast level [19]. Fundus camera captures the digital CFI without any injections into the blood vessels. Figure 6 shows the images captured with both techniques.

The contrasting agents enables retinal vessels appear brighter in invasive technique. The non-invasive techniques, though more secure, but it is tough to achieve same image quality as in the case of invasive techniques. The macula provides visual acuity and suffers from varying contrast. It is imperative to overcome this issue of contrast from macula region, because macula is the region that contained no blood vessels. Therefore, its observation plays a significant role in the early monitoring of related eye disease.

\section{Pathologies in RFIs}

RFIs can be used to screen and diagnose various pathologies. The DR severity levels or disease severity level are indicated through these diagnostics [21]. The following sections contained information of abnormalities in the fundus images.

\subsection{Microaneurysm in RFI}

The first clinical symptoms for early diagnosis of eyerelated illness are microaneurysm (MA). Referring to the CFI and FFA image in Figure 7, MAs emerge as tiny deep red lesions in CFI (white circles) are evidently seen as small round sharp-edged lesions with unevenly lighter rims [22]. In high-quality images, MA can be seen as small in scale, about $15-100 \mathrm{~m}$ in diameter. MAs are often somewhat oval, with relatively sharp edges with dark red dots. MAs have emerged as tiny blood vessel dilations and their appearances are associated with the development of DR [23]. These occur more in the area around macula.

\subsection{Blot and Dot Haemorrhages in RFIs}

A haemorrhage can also be diagnosed and detected with the help of RFIs. It can take any shape, be it round or irregular shapes. Haemorrhages are formed mostly due to the red cells. Tiny blots and dot are the types of haemorrhages. Blot haemorrhages mostly are larger than dot haemorrhages [21]. Figure 8 depicts the haemorrhages in fundus images.

\subsection{Soft Exudates \& Hard Exudates in RFIs}

Exudates can be seen as second clinical sign appearing after MA. Exudates are patches of random yellow or white colours found in the RFI with different dimensions, forms and at numerous locations [25]. The exudates are of two categories, i.e., the hard and soft exudates. Hard Exudates are produced by the serum lipoproteins which outpour from Micro-Aneurysms and dump in the retina [23]. The soft exudates are called cotton wool spots. Soft exudates exhibit bright prints where minuscale blood vessels are either blocked or damaged. Exudates are mostly fluffy lesions in fundus images and it affects the observation of blood vessels and in a case of FFA images, there is very difficult to observe the blood around the macula region due to exudates [26]. Figure 9 gives a description of the image containing the exudates.

\section{Severity Scale of Diabetic Retinopathy}

Important step for early diagnosis of the related eye disease is eye screening. For grading the DR, the standard grading protocol is used and it is elaborated in Table 1 [27][28]. It can be observed that with a boost in severity of DR, the facade of pathologies augments. The images of different severity scales are illustrated in Figure 10.

\section{Computerized Diagnosis for Diabetic Retinopathy Disease}

The computerized analysis of DR pathologies is increasing rapidly and the assessment of associated research is fractioned into three segments. The foremost one is associated to the automatic analysis of haemorrhages and microaneurysms, second one comprises of computerised analysis of exudates (both soft and hard) and lastly digital study of FAZ for classified the severity level of DR. Most researchers are focusing on the DR's computerised detection on the basis of pathologies. Microaneurysm can be identified automatically by early symptoms of DR, as stated by Kahai et. al. [29]. 


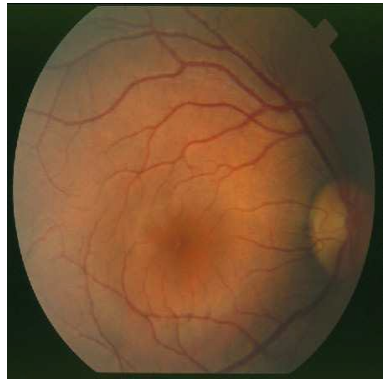

(a)

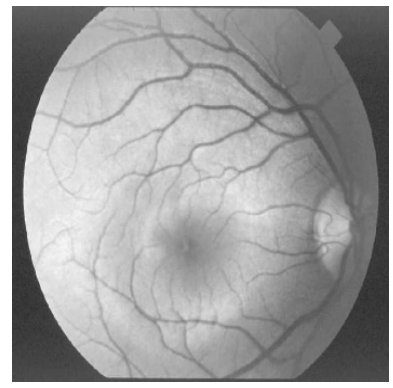

(b)

Fig. 5: Noise in fundus images [14] (a) RFI (b) CFIs green band

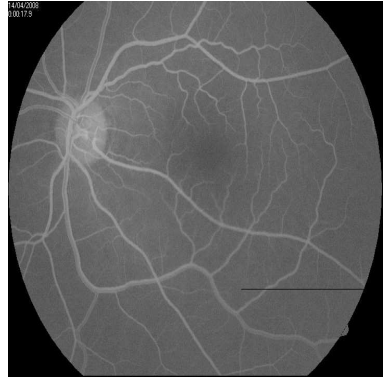

(a)

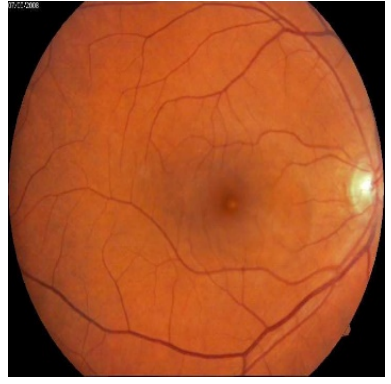

(b)

Fig. 6: FFA and digital CFI [20]

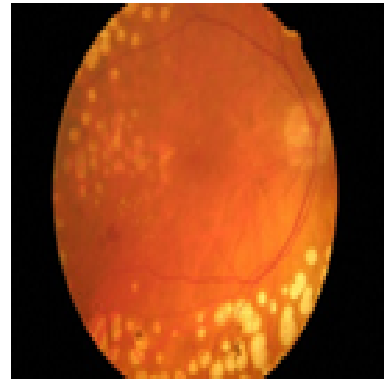

(a)

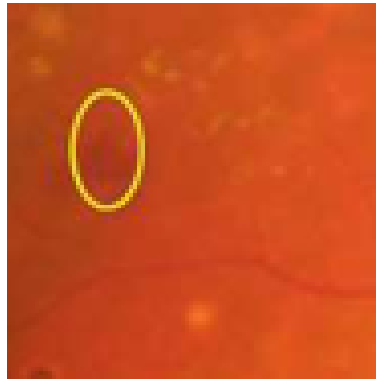

(b)

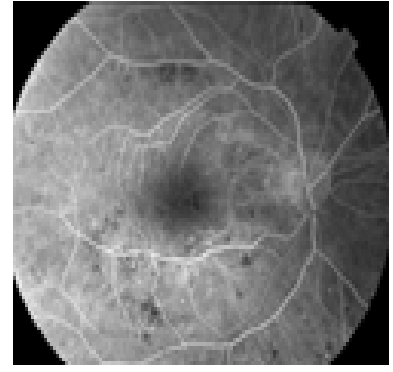

(c)

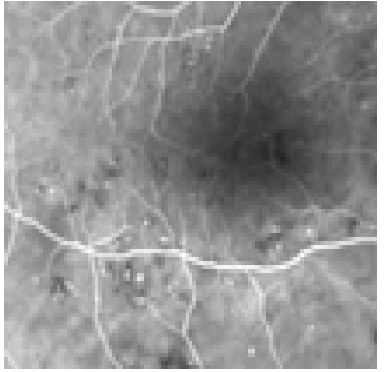

(d)

Fig. 7: Microaneurysm in CFI and FFA image: (a) illustration of CFI, (b) the macula region of colour fundus, (c) FFA image, (d) the macula region of FFA image

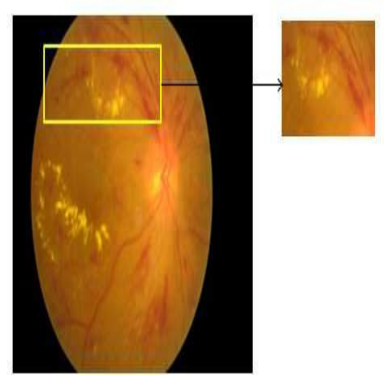

(a)

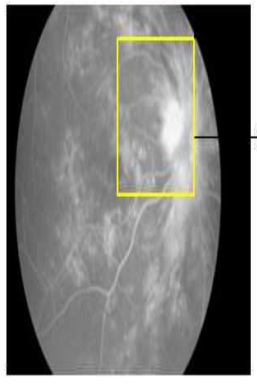

(b)

Fig. 8: FFA and digital CFI [20] 


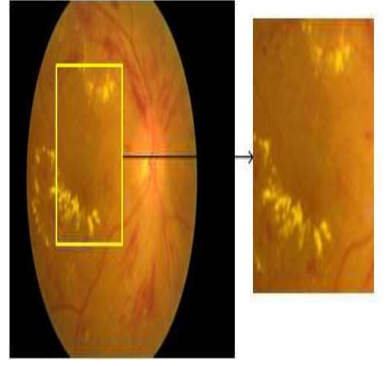

(a)

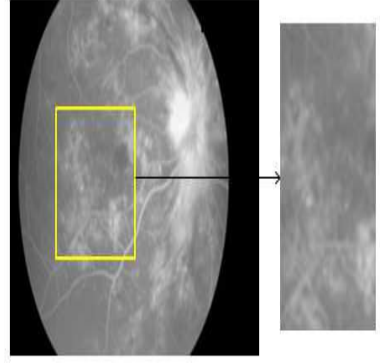

(b)

Fig. 9: Illustrations showing haemorrhages in CFI [2]; (a) CFI, (b) FFA Image

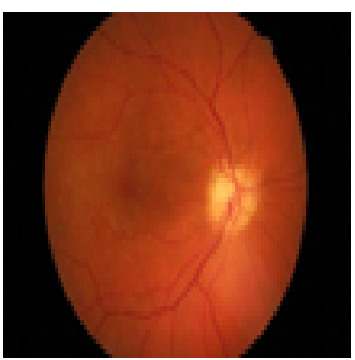

(a)

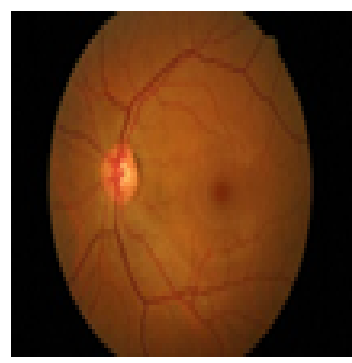

(b)

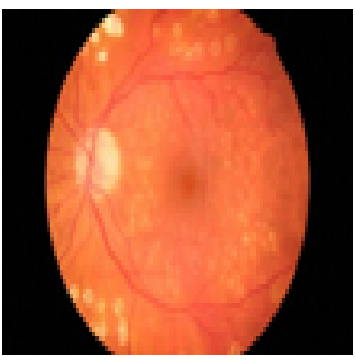

(c)

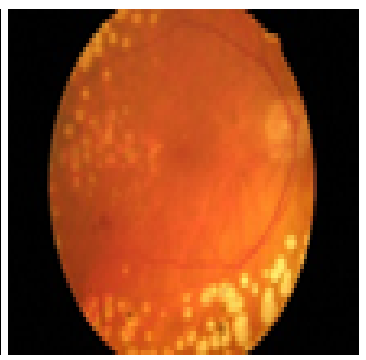

(d)

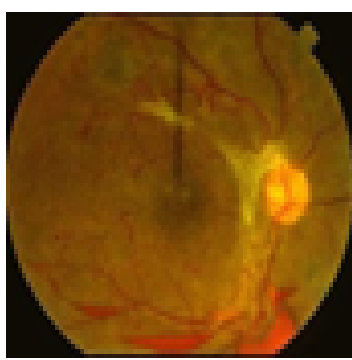

(e)

Fig. 10: Haemorrhages in CFI [2]; (a) absence of DR image, (b) NPDR image- Mild, (c) NPDR image- moderate, (d) NPDR image- severe, (e) PDR image

\begin{tabular}{|l|l|}
\hline Stages of DR & Criteria \\
\hline Absence of DR & $\begin{array}{l}\text { Usual images without any } \\
\text { abnormalities }\end{array}$ \\
\hline Slight NPDR & MAs only. \\
\hline Modest NNPDR & $\begin{array}{l}\text { Substantial MAs, hard/soft } \\
\text { exudates and Haemorrhages }\end{array}$ \\
\hline Extreme NPDR & $\begin{array}{l}\text { Any one of them. MAs in all } \\
\text { of four quadrants }\end{array}$ \\
\hline PDR & $\begin{array}{l}\text { Any one of them, Pre-retinal } \\
\text { Haemorrhages, Formation of novel } \\
\text { blood vessels at in a different place } \\
\text { (NVE) or disc (NVD). }\end{array}$ \\
\hline
\end{tabular}

TABLE 1: Grading scale of international DR [27][28]

Sinthanayothin et. al. [21] proposed an algorithms for detecting the number of exudates for early monitoring of DR. Later, another technique was suggested on the basis of detecting haemorrhages and microaneurysms by Hasen et. al. [31] and Larsen et. al. [30]. After them, a suitable method designed by Usher et. al. [32] for early detection of DR based on observing all three pathologies (microaneurysms, haemorrhages and exudates).

\subsection{Haemorrhage and Microaneurysm Based DR Detection}

Baudoin et. al. [33][2], on the basis of observing the microaneurysms from FFA images, proposed the auto- mated detection of associated eye diseases. Spencer et. al. [34] later employed the morphological methods, i.e., top hat transform in order to calculate patient's microaneurysms. In an extension to Baudoins research, utilization of match filtering joint operation technique, the microaneurysms of patients can be attained and top hat along with region growing. Another suitable method was projected by Walter et. al. [35] based on bounding box closing among top hat transform. As an alternative of utilizing a linear structural constituent with top hat transform, this method provided better results than Spencer et. al. [34]. For DR detection, apart from usage of morphological procedure, a novel technique to detect retinal pathologies based on the neural network was designed by Gardner et. al. [36]. Gardner's technique effectively worked on colour fundus image. Afterwards several techniques were proposed by various researchers [32][32][37]. The techniques of categorizing blood vessel network by using circular region increasing along with neural network were suggested by Usher et. al. [32].

Later on, Grisan et. al. [38] recommended a different novel technique with local spatial density based on group analogous pixels. Garcra et. al. [39] customized his own suggested method by utilising the mechanical feature variety and sorting steps. Another new 
idea was presented by Chutatape et. al. [40][41] to detect retinal microaneurysms on major component study. Later, Quellec et. al. [42][43] utilised template matching PCA to detect retinal microaneurysm. The efficiency of different techniques is elucidated in Table $2-4$.

\subsection{DR Detection Based on Hard and Soft Exu- date}

Initially, the hard and soft exudates were detected using thresholding technique. Wang et. al. [61] initiated the thresholding and shade correction method based semiautomatic exudate detection, but the thresholding requires user interaction. The previous structure was improved by Philips et. al. [62] and Zheng et. al. [63] with introduction of dynamic thresholding technique. The technique proposed by Philips et. al. [62] relies on the detection by global thresholding techniques of large, high-intensity areas of color-fundus image. Alternatively, the local threshold technique in blockwise order was used to section the smaller exudates.

The Philip's technique was a better way to detect pixels of exudate although provided numerous false positives. Zheng et. al. [63] implemented the local-neighboring exudate detection system alongside dynamic block-wise thresholding approach to resolve the false positive detection. The method for detecting exudates was suggested by Goldbaum et. al. [64]. Their technique was dependent on matching template alongside edge detection. This method was applied to image by positioning the benign lesion of image and this bright lesion was considered as exudates. Later, Goldbaum et. al. [64] customized its method by properly administered statistical pixel-based lesion arrangement rather than by using the template matching method to locate benign lesions.

Wang et. al. [61] used the related prototype along with the discriminating minimum distance classification to categorize the Benign Lesion in two categories, known as hard and soft exudates. The technique of identifying Benign Lesion as exudates using alternative approaches to the non-uniform correction factor for illumination was later enhanced by Sanchez et. al. [65]. Table 4 and 5 show the different methods recorded for detecting exudates.

\subsection{Foveal Avasculture Zone (FAZ) for Severe Level of Diabetic Retinopathy}

FAZ is one of the specific vision zones on the retina. It does not contain any blood vessels in its macula region [72]. Ibanez and Simo [73] were the first people who observed the FAZ in FFA images. It is found that progression of the disease increases due to an enlargement of the FAZ [74][75][76]. Due to hypertension and hemodynamic stress the tiny blood vessels around the FAZ possibly gets damage. It causes to blocked proper blood flow that affects the visual acuity [72]. The difficulty in graphic observation FAZ is that the patient can go through an oddly puffed-up FAZ in comparison to a patient with average vision [77].

The enlargement of FAZ has an effect that is apparent in colour-fundus images [78], which were previously seen in FFA images [77]. In the FFA image, the FAZ area has been determined utilising the Bayesian statistical techniques or thresholding methods dependent on the Sobel edge detectors as well as morphological operators [79]. The teams comprising H.A. Nugroho and Lila Izhar et. al. [78][80] worked on determining the FAZ area in CFI to overcome the usage of invasive technique. The method to study FAZ was developed by Lila Khar et. al. [80] relied on reconstruction and vessel extraction for DR grading and attained $92.2 \%$ accuracy.

\section{Retinal Fundus Image Ehancement Meth- ods}

Observation of fundus images is a challenging assignment due its noise along with varying low contrast problems. Without first resolving these problems, numerous researchers have contributed towards detecting DR. Since each retinal fundus image dataset has different problems, the enhancement procedure must provide the necessary application operations. To make a good upgrade, most medical images require contrast normalisation, contrast enhancement and noise removing processing of images. Owing to the variance of the light, contrast normalisation is a critical step for medical images, and small small in medical images are hard to distinguish. Therefore, methods of contrast enhancement have portrayed an vital role in enhancing the objects that are limited low-contrast. Noise impacts the quality of image, therefore, it is important to eliminate noise and gain more accurate image information in order to achieve improved image quality. Medical images for early disease diagnosis are analysed for clinical use such as retinal [6], MR [81], fluoroscopic [82], and microscopic images [83]. It is important to support doctors with a digital image processing system to accurately interpret image data. Because of these two challenges, the methodology of medical image enhancement can be divided into following three groups.

1) Normalization technique for different contrast medical images 


\begin{tabular}{|c|c|c|c|}
\hline Method & Sensitivity & Specificity & Database \\
\hline Quellec et. al. (2008) [42] & $90 \%$ & $90 \%$ & $120 \mathrm{FIs}$ \\
\hline Quellec et. al. (2008) [42] & $94 \%$ & $92 \%$ & $120 \mathrm{FIs}$ \\
\hline Quellec et. al. (2008) [42] & $90 \%$ & $89 \%$ & $120 \mathrm{FIs}$ \\
\hline Quellec et. al. (2006) [43] & $88 \%$ & $96 \%$ & $\begin{array}{l}\text { Manually Sectioned } \\
\text { FIs Database. }\end{array}$ \\
\hline Cree et. al. (1996) [44] & $82 \%$ & $84 \%$ & 20 FIs \\
\hline Frame et. al. (1998) [45] & $84 \%$ & $85 \%$ & 20 FIs \\
\hline Ege et. el. (2000) [46] & $69 \%$ & Not Provided & 134 FIs \\
\hline Hipwell et. al. (2000) [47] & $43 \%$ & Not Provided & 102 FIs \\
\hline Hipwell et. al. (2002) [47] & $81 \%$ & $93 \%$ & 102 FIs \\
\hline Yang et. al. (2001) [48] & $80 \%$ & $90 \%$ & 46 FIs \\
\hline Walter and Klein et. al. (2002) [49][70] & $86 \%$ & Not Provided & 05 FIs \\
\hline Walter et. al. (2002) [50] & $89 \%$ & Not Provided & 21 Annotate FIs \\
\hline Pallawala et. al. (2005) [51] & $93 \%$ & Not Provided & 102 FIs \\
\hline Fleming et. al. (2006) [52] & $85 \%$ & $83 \%$ & 71 FIs \\
\hline Bhalerao et. al. (2008) [53] & $83 \%$ & $80 \%$ & $\begin{array}{l}\text { DR Database } 1 \text { (DI- ARETDB1) } \\
\text { Database. }\end{array}$ \\
\hline Giri Babu Kande et. al. (2010) [54] & $100 \%$ & $91 \%$ & $\begin{array}{l}\text { DIARETDB1, Structured study } \\
\text { of the Retina (STARE) }\end{array}$ \\
\hline Zhang et. al. (2010) [55] & $71.30 \%$ & Not Provided & $\begin{array}{l}\text { DIARETDB1 Databases, Retinopathy } \\
\text { Online Challenge (ROC) }\end{array}$ \\
\hline Akara Sopharak et. al. (2011) [56] & $81.61 \%$ & $99.99 \%$ & 45 Non-dilated FIs \\
\hline Gowthaman et. al. (2014) [57] & $91.12 \%$ & $95.43 \%$ & $\begin{array}{l}\text { DIARETDB, Digital RIs for Vessel } \\
\text { Extraction (DRIVE) }\end{array}$ \\
\hline Raju S. Maher et. al. (2015) [58] & $94.86 \%$ & $95.6 \%$ & $\begin{array}{l}\text { DI- ARETDB0, (Diabetic Retinopathy } \\
\text { Database 0) }\end{array}$ \\
\hline Eftal Sehirli et. al. (2015) [59] & $69.1 \%$ & $99.3 \%$ & 113 FIs \\
\hline
\end{tabular}

TABLE 2: Existing microaneurysm automated detecting algorithms. Note: The images incorporated in this table are listed without the name of database indicating that authors use their own databases instead of the public databases available

\begin{tabular}{|l|l|l|l|}
\hline Method & Sensitivity & Specificity & Database \\
\hline Gardner et al (1996) [36] & 0.74 & 0.74 & ROC Database \\
\hline Zhang et al (2005) [40] & 0.90 & Not Provided & 35 FIs via Singapore National Eye Centre \\
\hline Ege et al (2000) [46] & 0.83 & Not Provided & 134 FIs \\
\hline Hatanaka et al (2008) [60] & 0.85 & 0.21 & A training dataset of 20 F. \\
\hline Hatanaka et al (2008) [60] & 0.80 & 0.80 & A training dataset of 125 FIs. \\
\hline
\end{tabular}

TABLE 3: Performance of accessible haemorrhages automated detection algorithms. Note: The images incorporated in this table are listed without database name indicating that own databases are used by authors instead of the public databases available

\begin{tabular}{|l|l|l|l|}
\hline Method & Sensitivity & Specificity & Database \\
\hline Niemeijer et al (2010) [1] & $30 \%$ & Not Provided & ROC database \\
\hline Sinthanayothin et al (2003) [16] & $78 \%$ & $89 \%$ & 771 FIs \\
\hline Grisan and Ruggeri et al (2007) [38] & $94 \%$ & Not Provided & A database consisting 60 annotated FIs \\
\hline Garcia et al (2008) [39] & $100 \%$ & $60 \%$ & 100 FIs \\
\hline Niemeijer et al (2000) [61] & $100 \%$ & $87 \%$ & 54 FIs \\
\hline
\end{tabular}

TABLE 4: Performances of accessible automated detecting algorithms for microaneurysm and haemorrhages. Note: The images incorporated in this table are listed without database name indicating that own databases are used by authors instead of the public databases available 


\begin{tabular}{|l|l|l|l|}
\hline Method & Sensitivity & Specificity & Database \\
\hline Zhang et al (2005) [40] & $88 \%$ & $84 \%$ & 30 Training FIs \\
\hline Ege et al (2010) [46] & $80 \%$ & Not Provided & 134 FIs \\
\hline Niemeijer et al (2007) [66] & $70 \%$ & $93 \%$ & 300 FIs \\
\hline Garcia et al (2008) [39] & $100 \%$ & $60 \%$ & 100 FIs \\
\hline Niemeijer et al (2000) [61] & $100 \%$ & $87 \%$ & 54 FIs \\
\hline
\end{tabular}

TABLE 5: Performance of soft exudates automated detecting algorithms. Note: The images incorporated in this table are listed without database name indicating that own databases are used by authors instead of the public databases available

\begin{tabular}{|l|l|l|l|}
\hline Method & Sensitivity & Specificity & Database \\
\hline Sinthanaya et al (2003) [16] & $89 \%$ & $100 \%$ & 771 FIs \\
\hline Gardner et al (1996) [36] & $93 \%$ & $93 \%$ & ROC Database. \\
\hline Ege et al (2000) [46] & $99 \%$ & Not Provided & 134 FIs \\
\hline Walter et al (2002) [49] & $93 \%$ & $92 \%$ & 21 Annotate FIs \\
\hline Phillips et al (1993) [62] & $87 \%$ & Not Provided & Not Reported. \\
\hline Osareh et al (2004) [67] & $90 \%$ & $89 \%$ & 67 FIs \\
\hline Osareh et al (2009) [68] & $94 \%$ & $92 \%$ & 300 FIs \\
\hline Sivaswamy and Ram et al (2009) [69] & $72 \%$ & Not Provided & Dataset - DIARETDB1 \\
\hline Ravishankar et al (2009) [70] & $95 \%$ & $91 \%$ & $\begin{array}{l}\text { STARE, DRIVE, Diaretdb0 } \\
\text { and Red Atlas databases. }\end{array}$ \\
\hline Xu and Luo et al (2009) [71] & $88 \%$ & $80 \%$ & $\begin{array}{l}\text { Database of 50 FIs - Beijing } \\
\text { Tongren Hospital }\end{array}$ \\
\hline
\end{tabular}

TABLE 6: Performances of accessible hard exudates automated detection algorithms. Note: The images incorporated in this table are listed without the name of database indicating that authors use their own databases instead of the public databases available

2) Image enhancement techniques for images with lower contrast

3) Image enhancement techniques for images with lower and different contrast

\subsection{Contrast Normalisation Techniques}

Normalization technique for varied contrast medical images are further categorised into following two types [84]: i) prospective contrast normalisation, and ii) retrospective contrast normalisation. For the modification of the varied contrast correction, prospective contrast normalisation methods need an acquisition procedure. It is also known as the technique of calibration. Whereas, in the processed image, retrospective forms depend on the specifics and make some conclusions about the image setting and can be used for the necessary applications [85]. In general, only the varied contrast trouble for system failures can be handled by practicable approaches. However, in retrospective approaches, most of the techniques for contrast normalisation are developed intensively. These are alluded to in Table 7.

\subsection{Techniques of Contrast Enhancement}

Techniques for image enhancement are classified in two classes for lower contrast images: i) choosing from either spatial domain image enhancement methods, and ii) frequency domain image enhancement techniques. Spatial domain methods are based on the process of contrast enhancement for modifying the histogram of the processed image and consequently and perform the process of enhancement in image's frequency domain [90]. Table 8 shows some of the widely used enhancement methods for low contrast images.

\subsection{Contrast Enhancement \& Normalisation Techniques}

The problem of low and varied contrast images could be dealt using the combination of image enhancement and image normalization techniques. Several researchers analysed these approaches on various medical images, but much of the work of the researchers involved enhancement techniques on the image of the retinal fundus or any medical image. At first, RFI necessitates to be normalised and later enhancement was done to solve the image's contrast problems. Toufique et. al. [95] proposed the method to overcome the varying low contrast issue. They applied their method to the retinal RFIs. A strong contrast enhancement factor of 5.38 was successfully obtained on retinal model images relative to the typical contrast enhancement factor on the FFA system at 5.79. However, improvement is still required for commercialized used. 


\begin{tabular}{|l|l|l|}
\hline Methods & Merits & Demerits \\
\hline Basis of Segmentation [81] & $\begin{array}{l}\text { Provide better contrast normalization } \\
\text { by choosing the classes of objects that } \\
\text { are explicitly modelled }\end{array}$ & $\begin{array}{l}\text { The assumption that a mixture of normal } \\
\text { distribution for a set of specifically modeled } \\
\text { classes achieves the distribution of image } \\
\text { intensity is mostly not valid for pathological } \\
\text { image data }\end{array}$ \\
\hline Filtering [86] & $\begin{array}{l}\text { Quick Execution. Speedy process and } \\
\text { well performed }\end{array}$ & Producing objects (formation of edge effects). \\
\hline Surface Fitting [87] & $\begin{array}{l}\text { Well executed if the image's homogeneous } \\
\text { areas are distinctive and broad }\end{array}$ & $\begin{array}{l}\text { During extrapolation, assessment of a varied } \\
\text { contrast region via concentrations of one main } \\
\text { region may source to some contrary information }\end{array}$ \\
\hline Statistical Model [88] & $\begin{array}{l}\text { In object classes no model is required for its } \\
\text { insensitivity to pathological data and completely } \\
\text { automated procedure }\end{array}$ & $\begin{array}{l}\text { Time of computation and efficiency of convergence } \\
\text { because of its iterative optimization }\end{array}$ \\
\hline Retinex [89] & $\begin{array}{l}\text { Increase image detail Increase image contrast } \\
\text { with even radiance and also minimize noise }\end{array}$ & The image size must be in the power of two \\
\hline
\end{tabular}

TABLE 7: Normalisation methods for varied contrast

\begin{tabular}{|c|c|c|}
\hline Methods & Merits & Demerits \\
\hline $\begin{array}{l}\text { Linear Contrast } \\
\text { Stretching methods }[90]\end{array}$ & $\begin{array}{l}\text { Well-performed improvement, simple } \\
\text { to execute, by extending those histogram } \\
\text { values but must be bimodal }\end{array}$ & $\begin{array}{l}\text { Due to the probability of getting different } \\
\text { values in the output image, the right value is } \\
\text { lost in the processed image }\end{array}$ \\
\hline Spatial Filtering [91] & $\begin{array}{l}\text { Using more local image information allows } \\
\text { image details to be enhanced }\end{array}$ & $\begin{array}{l}\text { Ringing objects and noise due to noise } \\
\text { enhancement and high contrast image } \\
\text { improvement }\end{array}$ \\
\hline $\begin{array}{l}\text { Global Histogram } \\
\text { Equalisation [91] }\end{array}$ & $\begin{array}{l}\text { Efficient for low contrast image enhancement } \\
\text { if one or two distinctive objects are included in } \\
\text { the image }\end{array}$ & $\begin{array}{l}\text { Enhanced image, resulting in unnecessary } \\
\text { visibility loss in objects with a high peak } \\
\text { in the histogram }\end{array}$ \\
\hline $\begin{array}{l}\text { Local Histogram } \\
\text { Equalisation }[92]\end{array}$ & $\begin{array}{l}\text { Improve the local contrast of the image and } \\
\text { produce more image detail }\end{array}$ & The creation of noise and objects \\
\hline $\begin{array}{l}\text { Wave-let based } \\
\text { Multi-Scale }[93]\end{array}$ & $\begin{array}{l}\text { Selectively improve or degrade image } \\
\text { characteristics of significance at various } \\
\text { levels of resolution }\end{array}$ & $\begin{array}{l}\text { The wave-let transform results are } \\
\text { not any longer shift invariant }\end{array}$ \\
\hline $\begin{array}{l}\text { Independent Component } \\
\text { Study (ICA) [94] }\end{array}$ & $\begin{array}{l}\text { ICA describes hidden variables called as } \\
\text { non-Gaussian and independent statistically } \\
\text { as the autonomous constituents of processed data }\end{array}$ & $\begin{array}{l}\text { The number of sources calculated is } \\
\text { not included in the order }\end{array}$ \\
\hline
\end{tabular}

TABLE 8: Enhancement methods for low contrast images

\section{Issues of Noise in Medical Images}

The variation in the brightness and colour information in the image is the indication of image noise. It most occurs due to the electronic circuitry of the digital camera. The Foremost objective of denoising is to improve the quality and maintain image details [96]. Before applying any denoising method, it is significant to know the nature of noise. There are many types of computer vision-based image processing techniques randing from linear image processing method to nonlinear image processing methods. There are different noise reduction schemes. Each of the schemes has its merits and demerits [97].

The characteristics of the noise in the medical image modality depend on the operating theory of the image modality, as defined in Figure 11. Medical images are seen in Figure 11. X-ray images are distorted by noise, hence the details of the image cannot be easily seen. Likewise, due to noise, ultrasound and
MRI images are impaired. The detection of such images is based on the origins of the devices' retrieval process. For instance, due to the intrinsic features of coherent imaging, an ultrasound image incorporates speckles or multiplicative noise. Noise occurred due to the performance of image sensors such as the digital camera is affected by illumination other environmental conditions during the acquisition process. Images obtained from CCD camera are affected due to light levels, sensors temperature and internal circuitry electronic noise [90].

\subsection{Effect of Noise in Retinal Fundus Images}

In the RFI with contained noise, as shown in Figure 11, it was tough to analysis blood vessel network. Especially, it is important to analyse blood vessel within the macula. The noise can also be found in green band. For the sake of observation, different regions of green band's image were cropped. The observation depicts 
that the blood vessels could not be seen clearly due to the noise. This discovery has shown that any improvement and extraction of the blood vessel procedure is compromised without reducing the noise from the RFI. A fundus camera has a complicated optical design and comprises two lighting mechanisms according to the basic feature of the fundus camera: the flash tube and the photo detector (camera circuitry) [98]. Due to the speckle flash or iteration of the patient's eye and a Fundus camera flash, noise in the RFI can be multiplicative. Due to the circuitry of the camera electronics, additive noise is also present since no digital image is a free addictive because it is recorded by cameras and noise is created by camera circuitry. Timothy et. al. [99] first clarified the study of the presence of noise in RFIs and also explain the effect of noise in fundus auto fluorescence imaging. The FFA image was processed by them and these data were collected by intrusive techniques. They only suggested the presence of noise, but did not clarify the existence of the noise in the FFA images. The literature shows various researchers have worked on RFI, but only focusing enhancement and detection in pathologies.

\subsection{Denoise Approaches for Medical Images}

Denoising is still one of the challenging issues because some techniques produce blurring and artefacts after denoising. Spatial filters are used which are either linear or non-linear filters. It is not possible to compute non-linear spatial filters only by using weighted sum. In order to calculate non-linear filters, various other operations such as square root, log, selection and sorting are applied. Median and weighted median filters are some examples of non-linear filters. In comparasion to the linear spatial filters such as non-linear Lee filter, Roberts filter, and Kirschs template filter, the application of non-linear filters is not easy. Compared to linear filters, non-linear filters can smooth with less blurring edges and can sense edges simultaneously at all orientations, but can be sluggish to compute. Any of the domain filtering transformations was directly used to suppress noise [100].

A signal can be made noise free by finding out a domain using transform domain filtering. There are three techniques of transform-domain filtering named as frequency transform, short frequency transform and wavelet transform that is utilised for the purpose of image denoising [100]. The frequency domain analysis can be analysed by using the Fourier transform analysis. The information of spectrum varied with respect to time cannot be provided by Fourier transform. To overcome such limitations, the Short Time
Fourier Transform (STFT) was proposed. It provides the signal observation with respect to both time and frequency. It contains a time window function and the window length is used to determine the time and frequency parameters. Hence, it requires a fixed time. In order to overcome this constraint, the wavelet transform was proposed for analysing the signals with different resolutions at different frequencies [101].

Chang et. al. [102] produced the novel method of de-noising by using the median filter with thresholding and their comparative study of other methods achieved a better performance. Folke et. al. [103] designed another approach of denoising based on combined operation of median and wavelet filters. However, it is one of the drawbacks of cascaded two filters for single task of noise removal. Smoothness in images was achieved by median filters [104] and objects are produced by wavelet. Owing to its high frequency coefficient, image detail vanishes because of smoothness of median filter [105][106].

Over the last decade, deionizing methods have been suggested on the subspace image structure. Among thes methods are the Least Squares (LS) and the Minimal Variance (MV) [107], which are based on the original image's Singular Values Decomposition (SVD) or covariance's Eigen Decomposition (ED). In order to minimise the distance between the signal subspace and the noisy image, they isolate the noise subspace from the signal subspace and use this information. At the risk of signal interference, the smallest squares and the minimum deviation gain substantial improvement in image de-noising. A novel subspace technique for monitor signal distortion as well as noise reduction has been proposed in recent times. This approach is an extension of the time-domain limit estimator of Van Trees et. al. [108] to two-dimensional signal constraints (image). The solution is suggested by Nidal Kamel et. al. [109].

Ephraim and Van have proposed the approach of signal subspace for the speech enhancement application. There was an extensive research work done on speech enhancement application and they introduced different techniques. The Time-Domain Constrained (TDC) estimators are one method is used for speech enhancement to handle noise and distortion level of the signals. The basic concepts of TDCE are dependent on the decomposition of noise subspace and signal subspace. It worked successfully to reduce the noise and control the signal distortion while minimised simultaneously [110]. Table 9 contains details of various denoising techniques explaining their strength and weakness. 


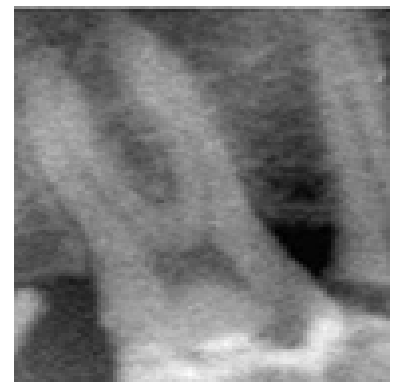

(a)

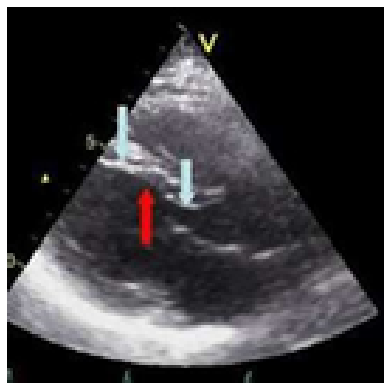

(b)

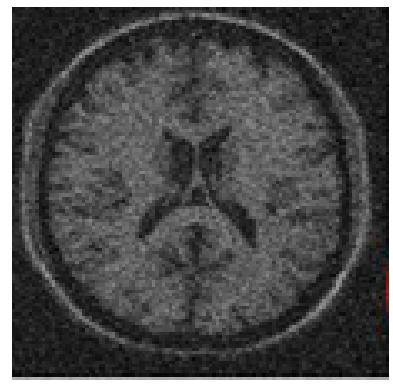

(c)

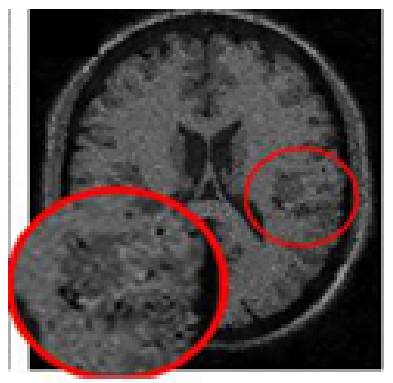

(d)

Fig. 11: Noise in the medical images [8]; (a) X-Ray, (b) ultrasound image, (c) MRI brain image

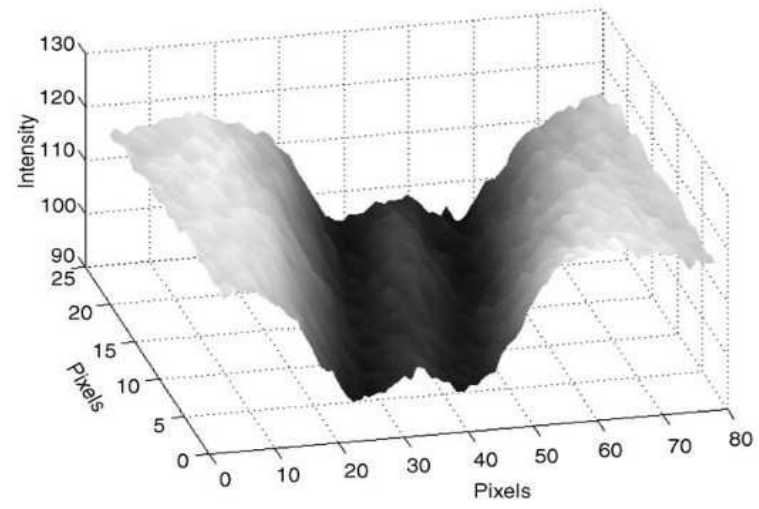

Fig. 12: An intensity profile of retinal blood vessel [117]

\section{Retinal Blood Vessel Segmentation}

For diagnosing of proper order, retinal vessels are the very important assessment for observation disease level. The retinal vascular network suffers from varying low contrast in between the RFI's background and blood vessels. Proper segmentation technique is required to maintain contrast amid the retinal blood vessels and background [114]. There are many techniques to section the retinal blood vessels. Some of them are elaborated below.

\subsection{Matched Filters for Vessel Detection}

The blood vessels segmentation through match filters mostly depends upon the two-dimensional linear structural elements and it is known as a kernel. Kernel has Gaussian-based derivative profile for identification of blood vessels cross section profile [115][116][117] (Figure 12). The kernel of the matched filter is rotated in a different orientation and usually it has 12 or 8 orientation to appropriate in the vessels of different structures. The image binarizations based on threshold are used to segment suitable vessels image. Accurate blood vessels are achieved through this concept, but the image of unhealthy or severe level DR patients does not provide fruitful results of segment blood vessels. The tram line filters are non-linear filters that are proposed to achieve the accurate results of blood vessel segmentation either on healthy images or unhealthy images [118]. Using running kernel as structuring elements causes difficulty of fitting the pathologies and mostly twisting blood vessels. The match filters do not produce any distortion, but they require the binary thresholds identifying the vessels and non-vessels of the image.

\subsection{Vessel Tracking for Retinal Vessel}

Neural network was one method used for segment retinal vessels and it gave better results as compared to other proposed method [123]. Initially, the neural network is proposed for detection of the edge of retinal image and PCA was applied as pre-processing steps in order to manage the image's background noise [124]. Sinthanayothin et. al. [124] proposed techniques of detection of blood vessels and their method provided a success rate of $99.56 \%$ on the trained data and $96.88 \%$ on the validation data with an overall performance of $83.3 \%$ sensitivity and $91 \%$ of specificity.

\subsection{Neural Networks}

The neural network was one method used for segment retinal vessels and it gave better results as compared to other proposed method [123]. Initially, the neural network is proposed for detection of the edge of retinal image and PCA was applied as pre-processing steps in order to manage the image's background noise [124]. Sinthanayothin et. al. [124] proposed a technique for the detection of blood vessels and their method provided the success rate of $99.56 \%$ on the trained data and $96.88 \%$ on the validation data with an overall performance of $83.3 \%$ sensitivity and $91 \%$ specificity.

\subsection{Morphological Processing}

Blood vessel segmentation is not an uphill task, but it is a really good work to detect accurate and proper 


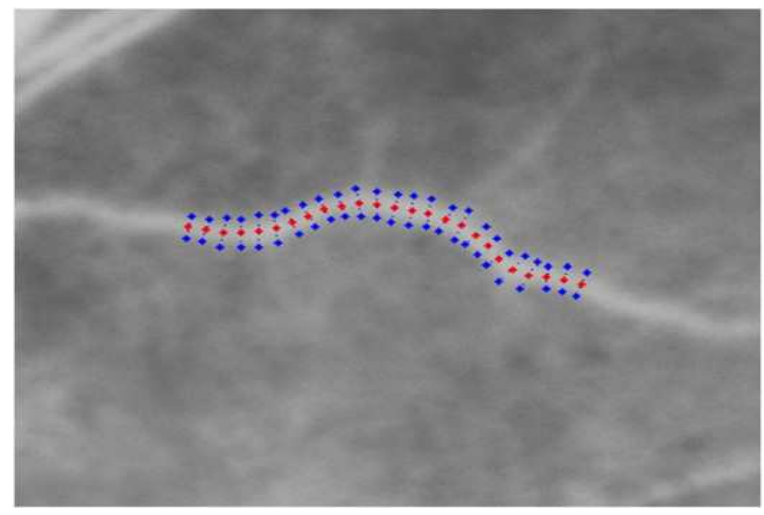

Fig. 13: Tracking process of retinal vessel grey scale image [119]

shape blood vessel. The morphological image processing techniques are playing an imperative role in the detection of perfect blood vessels in its good form. There are two types of structural elements that are used in morphological image processing techniques: i) added (it is known as dilation morphological, structural element), and ii) subtract (it is known as erosion morphological, structural element). To maintain the proper shapes of the image vessels, opening and closing morphological techniques are utilized for detection of proper forms of retinal blood vessels [125][126]. The Table 10 illustrated different technique for blood vessels extraction.

\section{Automated Detection of Diabetic Retinopathy}

There is a rapid development in computerised detection system of DR and other eye related disease over last three decades [141]. Lee et. al. [142] invented the computerized system for primary analysis of the red retinal injury and evaluated system results against FFA images. Following subsection explains the different computerised detection system for related eye diseases. The study of DR reported that $3.6 \%$ of youngsters suffering from type- 1 diabetes, and $1.6 \%$ of old people suffering from type-2 diabetes were reported as legal blind [141]. Figure 10 showed the different pathologies and other alteration of veins in retinal image. The key pathologies of DR are haemorrhages, hard and soft exudates, microaneurysm, and blood vessels in macula region [143][144][145].

\subsection{Detection Methods of DR}

The Bayesian statistical method is utilized in the DR images for identification of lesion and non-lesion to categorize the lesion and non-lesion pixels [61]. Wang et. al. [61] achieved an outstanding 100\% accuracy in detecting the lesion in irregular retinal images and precision of $70 \%$ in ordinary retinal images [61]. The neural network multilayer technique has been used to categorize typical retinal images and other phases of DR images [16]. Samuel et. al. [146] provided $80.21 \%$ sensitivity and $70.66 \%$ sensitivity to the lesion and non-lesion identification system dealing with DR images. In terms of exactitude, it was their system's notable achievement that $81.7 \%$ results are attained on the server and PDR phases of DR images.

A method proposed by Singalavanija et. al. [147] for the investigation of DR images for detection of exudates, microaneurysms and haemorrhages gave a sensitivity of $74.8 \%$ and precision of $82.7 \%$. This method introduced a novel definition of grading DR by differentiating it and safe retinal images. AfterwardsKahai et. al. [148] suggested a DR monitoring and decision support system which was utilized alongside Bayes optimal classification method to classify pathologies, particularly microaneurysms. This was a successful method in DR grading with sensitivity of $100 \%$ and specificity of $67 \%$. Wang et. al. [149] suggested a different early-detection approach for DR and used the categorization of DR images into levels. A neural network had been used to assess disease progression. $90 \%$ sensitivity, $100 \%$ precision with $84 \%$ accuracy was achieved through their process. A new technique for identifying stages of DR was given by Nayak et. al. [150] and their neural network-based technique to determine the texture parameters of the retinal image part of the exudates and the blood vessels. They achieved $93 \%$ accuracy with sensitivity of $90 \%$ and a $100 \%$ specificity.

The vector support mechanism was used by Acharya et. al. [151] to recognize the DR stages and achieved accuracy and sensitivity of $82 \%$, and $88 \%$ specificity. Their method to train data for validation purposes and the program was able to categorize the unidentified classified data into different stages of DR with an $82 \%$ accuracy, $82 \%$ sensitivity and $86 \%$ precision. Nicolai et. al. [152] presented the screening method of DR and validated their program with a number of patients suffering from any stage of DR. Usher et. al. [32] subsequently suggested another DR screening method and obtained a higher patient-based sensitivity of $95.1 \%$ of DR detection with a precision of 46.3\%. Neubauer et. al. [153] suggested a DR screening system reliant on optical disk and retinal thickness analysis. Their system achieved a sensitivity of $93 \%$ at stages of PDR together with all stages, and a specificity of $100 \%$ in other situations.

Lee et. al. [154] suggested a software-based DR 


\begin{tabular}{|l|l|l|}
\hline Methods of Denoising & Merits & Demerits \\
\hline Mean Filter & Easy implementation & $\begin{array}{l}\text { Loss of image data due } \\
\text { to image blurring }\end{array}$ \\
\hline Median Filter [17] & Easy implementation & $\begin{array}{l}\text { Lose the image details } \\
\text { due to image blurring }\end{array}$ \\
\hline Wiener Filter [111] & $\begin{array}{l}\text { Local filtering is carried out } \\
\text { and the noise is eliminated while } \\
\text { still retaining the contrast }\end{array}$ & $\begin{array}{l}\text { The Wiener filter makes } \\
\text { the image smooth and image } \\
\text { information are lost due to } \\
\text { its smoothness }\end{array}$ \\
\hline Wave-let Transform [112] & $\begin{array}{l}\text { In both time and frequency, } \\
\text { signals can be detected. }\end{array}$ & $\begin{array}{l}\text { Generate objects. The results } \\
\text { of the transformation of wavelets } \\
\text { are no longer invariant changes }\end{array}$ \\
\hline Least Squares Estimator (LSE) [105] $]$ & $\begin{array}{l}\text { Offer more appropriate results of } \\
\text { noise reduction compared to the } \\
\text { domain of Spatial and Transform }\end{array}$ & $\begin{array}{l}\text { The LSE cannot control the } \\
\text { distortion of signals }\end{array}$ \\
\hline $\begin{array}{l}\text { The Minimum Variance } \\
\text { Estimator (MVE) [113] }\end{array}$ & $\begin{array}{l}\text { Far greater control when combined } \\
\text { with LSE over signal distortion }\end{array}$ & $\begin{array}{l}\text { MVE can't control the } \\
\text { distortion of the signal }\end{array}$ \\
\hline
\end{tabular}

TABLE 9: Comparison among image denoising methods

grading system and a method of classifying DR by three kind of lesion, i.e., haemorrhages, microaneurysms, and exudates. In this method, two datasets were used out of which one included 430 images. Their device recognizes haemorrhages with $82.6 \%$ accuracy, microaneurysms with $82.6 \%$ accuracy, and exudates with $88.3 \%$ accuracy. Certain sets held for $85.3 \%$ haemorrhage recognition and $87.5 \%$ and $93.1 \%$ of microaneurysms and exudates on 361 images. Philip et. al. [62] suggested another DR screening method. Their classification was focused on illness with no disease detection resulting in sensitivity of $90.5 \%$ and specificity of $67.4 \%$. Estabridis et. al. [155] suggested a DR screening mechanism reliant on the identification of specifications, i.e., FAZ, retinal vascular network and pathologies. They achieved $90 \%$ accuracy. Li et. al. [156] proposed a screening method DR using color RFIs with sensitivity of $80.5 \%$ and $90.8 \%$ positive prediction values on all DR stages. Abramoff et. al. [157] evaluated the screening of the proposed DR system with a sensitivity of $84 \%$ and a specificity of $64 \%$ on the very large amount of database. A support machine classifier along with its higher order spectra features was used to classify the stages of DR with $82 \%$ accuracy. Vujosevic et. al. [158] suggested a single pathology detection method for the grading of clinical DR and stages of PDR from color fundus images with $82.1 \%$ sensitivity and $92.5 \%$ specificity for DR grading.

\section{Conclusion \& Future Work}

Continuous diabetes forms the structure of various pathologies, but in majority of cases, first microaneurysms were formed and then exudates as well as haemorrhages followed. The significant significance to prevent vision loss is earliest detection of DR. Early detection can be detected easily by microaneurysm identification and the FAZ field analysis. This review gave a detailed description of different research areas related to the analysis of related eye diseases from RFIs. We explained different image enhancements methods along with their merits and demerits. Our analysis will help the researchers to select the suitable image enhancement methods. We also explained various deionising methods along with their strengths and weaknesses which will help researchers to select suitable denoising methods for medical images as fundus images and general images also. In addition, we also explained different methods of detection of blood vessels for early detection of related eye disease. Many researchers work on vessel extraction from fundus images; we described each method along with their performance and limitation. Many researchers will get help from this review for proposing a novel method for retinal blood vessel extraction.

According to the crucial review of the abovementioned specific research areas related to color fundus image analysis, it is obvious that a fully automatic DR grading system which gives the most accurate detection results is required. In this regard, it is initially important to use fully automatic and novel methods to detect blood vessels, even the tiny blood vessels, to improve the efficiency of the automatic mechanism. As we explained, blood vessel identification provides the indication of progression of the disease since DR progresses with vessel variability.

Another purpose of this study is to analyse different algorithms for automatic detection which is an important requirement to improve or develop robust algorithms for DR monitoring at the premature stage, as early detection of eye diseases can be helpful to save 
vision.

\section{References}

[1] Niemeijer M, Van Ginneken B, Cree MJ, Mizutani A, Quellec G, Sánchez CI, Zhang B, Hornero R, Lamard M, Muramatsu C, Wu X., "Retinopathy onlinechallenge: Automatic detection of microaneurysms in digital color fundus photographs," IEEE Transactions on Medical Imaging, vol. 29, no. 1, pp. 185-95, 2010.

[2] Faust, Oliver, Rajendra Acharya, Eddie Yin-Kwee Ng, Kwan-Hoong Ng, and Jasjit S. Suri. "Algorithms for the automated detection of diabetic retinopathy using digital fundus images: a review." Journal of medical systems, vol. 36, no. 1, pp. 145-157, 2012.

[3] L. M. Ruta, Magliano DJ, Lemesurier R, Taylor HR, Zimmet PZ, and Shaw JE., "Prevalence of diabetic retinopathy in type 2 diabetes in developing and developed countries," Diabet Med,vol. 30, no. 4, pp. 387-98, 2013.

[4] P. D.Malaysia, "Clinical practice guidelines (cpg) management of type 2 diabetes mellitus," Ministry of Health Malaysia, Malaysian Endocrine and Metabolic Society, Academy of Medicine Malaysia, vol.1, pp. 1000, 2009.

[5] Ahmad Fadzil, L. I. Izhar, and H. A. Nugroho, "Determination of foveal avascular zone in diabetic retinopathy digital fundus images," Computers in Biology and Medicine, vol. 40, no. 7, pp.657664, 2010.

[6] M.Fadzil, H. A. Nugroho, H. Nugroho, and I. L. Iznita, "Determination of retinal pigments from fundus images using independent component analysis," International Conference on DigitalImage Processing,, vol. 1, pp. 137 - 141, 2008.

[7] M. Nair and Nair UP, "Digital and advanced imaging in endodontics: A review," Journal of Endodontics, vol. 33, no. 1, pp.1-6, 2007.

[8] U. Vovk, F. Pernus, and B. Likar, "A review of methods for correction of intensity inhomogeneity in mri," Medical Imag-ing,IEEE Transactions „, vol. 26, no. 3, pp. $405-421$, 2007.

[9] Ryan Phillips, "What to wear during fluoroscopy," Phillips SafetyProduct, 2013.

[10] The Worlds of David Darling, "Computed tomography (ct)scanning," Encyclopedia of Alternative Energy, 2016.

[11] Positron Emission Tomography (PET) Chillers, "Positron emission tomography (pet)," Medical Chillers, 2015

[12] America Pink, "Single-photon emission computed tomogra-phy,"Websie of America pink, 2016.

[13] A Todd, Gould, , and Molly Edmonds, "How mri works," Science How the Stuff works, 2016.

[14] Toufique Ahmed Soomro, "Non-invasive image denoising and contrast enhancement techniques for retinal fundus images," M.S. thesis, Electrical and Electronic Engineering Department Universititeknologi Petronas, 2014.

[15] Michelson E. Peli, "Contrast in complex images," Journal of theOptical Society of America, vol. 7, no. 10, pp. 2032-40, 1990.

[16] C. Sinthanayothin, V. Kongbunkiat, S. Phoojaruenchanachai, and A. Singalavanija, "Automated screening system for diabetic retinopathy," The 3rd International Symposium on Image and SignalProcessing and Analysis, vol. 2, pp. $915-920,2003$.

[17] M. Salem and A. K. Nandi, "Novel and adaptive contribution of the red channel in pre-processing of colour fundus images,"Journal of the Franklin Institute, vol. 344, no. 3, pp. 243256, 2007.
[18] Ahmad Fadzil M Hani, Toufique Ahmed Soomro, Ibrahima Faye, Nidal Kamel, and Norashikin Yahya, "Identification of noise in the fundus images," IEEE International Conference on Control System, Computing and Engineering, vol. 1, pp. $191-196,2013$.

[19] G. Zahlmann, B. Kochner, D. Schuhmann, B. Liesenfeld, andA. Wegner, "Hybrid fuzzy image processing for situation assessment diabetic retinopathy," IEEE Engineering in Medicine andBiology Magazine, vol. 19, pp. 76 - 83, 2000.

[20] Shirin Hajeb Mohammad Alipour, Hossein Rabbani, Moham-madreza Akhlaghi, Alireza Mehri Dehnavi, and ShaghayeghHaghjooyJavanmard, "Analysis of foveal avascular zone for grading of diabetic retinopathy severity based on curvelet transform.," Graefes Arch Clin Exp Ophthalmol, vol. 250,no. 11, pp. 1607-14, 2012.

[21] C. Sinthanayothin and J. Boyce, "Automated detection of diabetic retinopathy on digital fundus images," Diabetic Medicine, vol. 19,no. 2, pp. 105-12, 2002.

[22] M. Niemeijer and B. van Ginneken, "Automatic detection of red lesions in digital color fundus photographs," IEEE transactionson Medical Imaging, vol. 24, no. 5, pp. 584-92, 2005.

[23] G. Matsopoulos, N. Mouravliansky, K. K. Delibasis, and K. S. Nikita, "Automatic retinal image registration scheme using global optimization techniques," IEEE Transactions on InformationTechnology in Biomedicine, vol. 3, no. 1, pp. 47-60, 1999.

[24] T.Wu, "Review of diabetes: Identification of markers for early detection, glycemic control, and monitoring clinical complications,"Journal of Clinical Laboratory Analysis, vol. 7, pp. 293-300, 1993.

[25] W. Huan, W. Hsu, G. Kheng Guan, and L. Mong Li, "An effective approach to detect lesions in color retinal images," IEEE Conference on Computer Vision and Pattern Recognition, vol. 2, pp. $181-186,2000$.

[26] http://medweb.bham.ac.uk/easdec/eyetextbook/dminternet.htm

[27] N. Collins, "Diabetic retinopathy preferred practice pattern,"American Academy of opthhalmology, 2003.

[28] M. Herbert and F.Jelinek, "Automated image detection of retinal pathology," CRC Press Taylor and Francis Group, vol. 1, pp. 384,2010.

[29] P. Kahai, K. Namuduri, and H. Thompson, "Decision support for automated screening of diabetic retinopathy," the Thirty-Eighth Asilomar Conference on Signals, Systems and Computers, vol. 1, pp.7 pages, 2004.

[30] G. Larsen, JannikGodtamd Michael Grunkinamd Henrik Lund- Andersen, andMichael Larsen, "Automated detection of diabetic retinopathy in a fundus photographic screeningpopulation," In-vest Ophthalmol Vis Sci., vol. 44, no. 2, pp. 767-771, 2003.

[31] A Hansen, Hartvig, Jensen MS, Borch-Johnsen, LundAndersen H, and Larsen M., "Diabetic retinopathy screening using digital non-mydriatic fundus photography and automated image analysis," Acta Ophthalmologica Scandinavica, vol. 82, no. 6, pp. 666-72,2004.

[32] D. Usher and Dumsky, "Automated detection of diabetic retinopathy in digital retinal images: a tool for diabetic retinopathyscreening," Diabetic Medicine, vol. 21, no. 84-90., pp. 1, 2003.

[33] Baudoin, Klein JC, and Jean-Claude Klein, "Automatic detection of microaneurysms in diabetic fluorescein angiography," Computers And Biomedical Research, vol. 32, no. 3, pp. 254-61, 1996.

[34] T. Spencer, Olson, Ken Mchardy, Peter F. Sharp, and John V. Forrester, "An image-processing strategy for the segmentation and quantification of microaneurysms in flu- 
orescein angiogramsof the ocular fundus," Computers And Biomedical Research, vol. 29,no. 4, pp. 284-302, 1996.

[35] T. Walter and J. C. Klein, "A contribution of image processingto the diagnosis of diabetic retinopathy-detection of exudates incolor fundus images of the human retina," IEEE Transactions onMedical Imaging, vol. 10, pp. 1236-43., 2002.

[36] G. Gardner, Keating, G G Gardner, D Keating, T H William-son, and A T Elliott, "Automatic detection of diabetic retinopathyusing an artificial neural network: a screening tool," BritishJournal of Ophthalmology, vol. 80, no. 11, pp. 940944, 1996.

[37] G. Luo, OpasChutatape, Huiqi Li, and S. M. Krishnan, "Ab-normalitydetection in automated mass screening system of diabeticretinopathy," Proceedings of the IEEE Symposium on Computer-Based Medical Systems (CBMS), vol. 1, pp. $132-137,2001$.

[38] A. Enrico Grisan and Ruggeri A, "Segmentation of candidatedark lesions in fundus images based on local thresholding andpixel density," The 29th Annual International Conference of the EEEEngineering in Medicine and Biology Society (EMBS), vol. 1, pp.6736-9., 2007.

[39] M. Garcia, Sanchez CI, Lopez MI, Diez A, and Hornero R.,"Automatic detection of red lesions in retinal images using amultilayer perceptron neural network," The Annual InternationalConference of the IEEE Engineering in Medicine and Biology Society(EMBS), vol. 10, pp. 5425-8, 2008.

[40] X. Zhang and Chutatape, "A svm approach for detection of hemorrhagesin background diabetic retinopathy," IEEE InternationalJoint Conference on Neural Networks, vol. 4, pp. $2435-2440,2005$.

[41] X. Zhang and O. Chutatape, "Top-down and bottomup strategiesin lesion detection of background diabetic retinopa-thy,"IEEE Computer Society Conference on Computer Vision and PatternRecognition, vol. 2, pp. $422-428$, 2005.

[42] G. Quellec and Lamard, "Optimal wavelet transform for thedetection of microaneurysms in retina photographs," IEEE Transactions On Medical Imaging, vol. 27, no. 9, pp. 1230-41, 2008.

[43] G. Quellec and Lamard, "Detection of lesions in retina photographsbased on the wavelet transform," The 28th AnnualInternational Conference of the IEEE Engineering in Medicine andBiology Society (EMBS), vol. 1, pp. 2618-21, 2006.

[44] J. Cree, J. A. Olsoni, K. C. McHardyt, J. V. Forresters, and P. F.Sharp, "Automated microaneurysm detection," Interna-tionalConference on Image Processing, vol. 1, pp. 700-702, 1996.

[45] Frame, Peter E. Undrilla, Michael J. Creea, John A. Olsonb,Kenneth C. McHardyc, Peter F. Sharpa, and John V. Forresterb,"A comparison of computer based classification methods appliedto the detection of microaneurysms in ophthalmic fluoresceinangiograms," Computers in Biology and Medicine, vol. 28, no. 3,pp. 225238, 1998.

[46] B. Ege, Ole K. Hejlesena, Ole V. Larsena, Karina M?llera, BarryJenningsb, David Kerrb, and David A. Cavanb, "Screening fordiabetic retinopathy using computer based image analysis andstatistical classification," Comput Methods Programs Biomed, vol.62, no. 3, pp. 165175, 2000.

[47] Hipwell, Strachan F, Olson JA, McHardy KC, Sharp $\mathrm{PF}$, and-Forrester JV, "Automated detection of microaneurysms in digital red-free photographs: a diabetic retinopathy screening tool," Diabetic Medicine, vol. 17, no. 8, pp. 588-94, 2000.
[48] G. Yang, L. Gagnonz, S. Wangy, and M.C. Boucher, "Algorithm for detecting micro-aneurysms in low-resolution color retinal images," In Proceedings of Vision Interfaces (VI), vol. 4, pp. 265-271., 2001.

[49] T. Walter and Klein, "Automatic detection of microaneurysms in color fundus images of the human retina by means of the bounding box closing," In Medical Data Analysis, vol. 2526, pp.210-220, 2002.

[50] T.Walter and Jean-Claude Klein, "Automatic detection of microaneurysms in color fundus images of the human retina by means of the bounding box closing," Springer Medical Data Analysis, vol.2525, pp. 220-230, 2002.

[51] P. Pallawala, W. Hsu, M. L. Lee, and S. S. Goh, "Automatedmicroaneurysm segmentation and detection using generalizedeigenvectors," Seventh IEEE Workshops on Application of ComputerVision (WACV/MOTION), vol. 5, no. 1 , pp. $322-327,2005$.

[52] Fleming, S. Philip, K. A. Goatman, and J. A. Olson, "Automatedmicroaneurysm detection using local contrast normalization andlocal vessel detection.," IEEE Transactions On Medical Imaging,vol. 25, no. 9, pp. 1223 - 1232, 2006.

[53] A. Bhalerao, A. Patanaik, S. Anand, and P. Saravanan, "Ro-bustdetection of microaneurysms for sight threatening reti-nopathyscreening," The 2008 Sixth Indian Conference on Computer Vision, Graphics \& Image Processing, vol. 1, pp. $520-527,2008$.

[54] Giri Babu Kande, T Satya Savithri, and P Venkata Subbaiah, "Automatic detection of microaneurysms and hemorrhages indigital fundus images," Journal of digital imaging, vol. 23, no. 4,pp. 430-437, 2010.

[55] B.Zhang, X.Wu, J.You, Q.Li, and F.Karray, "Detection of microaneurysmsusing multi-scale correlation coefficients," Pattern-Recognition, vol. 43, no. 6, pp. 22372248., 2010.

[56] AkaraSopharak, BunyaritUyyanonvara, Sarah Barman, and TomWilliamson, "Automatic microaneurysm detection from nondilateddiabetic retinopathy retinal images," Proceedings of theWorld Congress on Engineering WCE London, U.K., vol. 2, pp. 4pages, 2011.

[57] Gowthaman, "Automatic identification and classification ofmicroaneurysms for detection of diabetic retinopathy," IJRET:International Journal of Research in Engineering and Technology, 2014.

[58] Raju S. Maher, Babasaheb Ambedkar, Sangramsing N. Kay-te,Babasaheb Ambedkar, Mukta Dhopeshwarkar, and Ba-basahebAmbedkar, "Evaluation of a system for automatic detection ofdiabetic retinopathy from color fundus photographs for screeningpopulation," International Journal of Computer Applications,vol. 131, no. 3, pp. 0975 8887, 2015.

[59] EftalSehirli, Muhammed Kamil Turan, and Alexander Diet-zel,"Automatic detection of microaneurysms in rgb retinal fundusimages," International Journal of Scientific and Technological Research, vol. 1, no. 8, pp. 1-7, 2015.

[60] Y. Hatanaka, Nakagawa T, Hayashi Y, and Hara T andFujita H., "Improvement of automated detection method of hemorrhagesin fundus images," 30th Annual International Conference of theIEEE Engineering in Medicine and Biology Society (EMBS), vol. 1,pp. 5429-32., 2008.

[61] H. Wang, Wynne Hsu, Kheng Guan Goh, and Mong Li Lee, "An effective approach to detect lesions in color retinal images,"IEEE Computer Society Conference on Computer Vision and PatternRecognition (CVPR), vol. 2, pp. 181 $186,2000$.

[62] R. Phillips and Forrester, "Automated detection and quantificationof retinal exudates," Graefes Archive for Clinical 
andExperimental Ophthalmology, vol. 231, no. 2, pp. 90-94, 1993.

[63] L. Zheng and Chutatape, "Automatic image analysis of fundusphotograph," 19th Annual International Conference of the IEEEEngineering in Medicine and Biology Society, vol. 2 , pp. $524-525,1997$.

[64] M. Goldbaum, Katz NP, Nelson MR, and Haff LR., "The discriminationof similarly colored objects in computer images of theocular fundus," Investigative Ophthalmology \& Visual Science, vol.31, no. 4, pp. 617-23, 1990.

[65] Sanchez, Garc?a M, Mayo A, Lopez MI, and Hornero R, "Retinalimage analysis based on mixture models to detect hard exudates,"Medical Image Analysis, vol. 13, no. 4, pp. 650-8, 2009.

[66] M. Niemeijer, Van Ginneken B, Russell SR, SuttorpSchulten MS,and Abramoff MD., "Automated detection and differentiation ofdrusen, exudates, and cotton-wool spots in digital color fundusphotographs for diabetic retinopathy diagnosis," InvestigativeOphthalmology and Visual Science, vol. 48, no. 5, pp. 2260-7, 2007.

[67] A. Osareh, "Automated identification of diabetic retinal exudatesand the optic disc," hD thesis, Department of Computer Sciene,University of Bristol, vol. 1, pp. 300, 2004.

[68] A. Osareh, BitaShadgar, and Richard Markham, "Acomputational-intelligencebased approach for detection of exudatesin diabetic retinopathy images," IEEE Transactions OnInformation Technology In Biomedicine, vol. 13, no. 4, pp. 535-545,2009.

[69] K. Ram and Sivaswamy, "Multi-space clustering for segmentationof exudates in retinal color photographs," Annual Interna-tional Conference of the IEEE Engineering in Medicine and BiologySociety (EMBS), vol. 1, pp. 1437-40, 2009.

[70] Ravishankar, A. Jain, and A. Mittal, "Automated feature extractionfor early detection of diabetic retinopathy in fundus images,"In Proceedings of the IEEE Computer Society Conference on ComputerVision and Pattern Recognition (CVPR), vol. 1, pp. 210-217, 2009.

[71] Lili Xu and Shuqian Luo, "Support vectormachine based methodfor identifying hard exudates in retinal images," In Proceedings ofthe IEEE Youth Conference on Information, Computing and Telecommunication (YC-ICT), vol. 1, pp. $138-141,2009$.

[72] G. Patrick, "fluorescein and icg angiography," Thieme medi-calpublisher, vol. 1, pp. 500, 2008.

[73] M. Ibanez and A. Simo, "Bayesian detection of the fovea in eyefundus angiographies," Pattern Recognition Letters, vol. 20, no. 2,pp. 229240, 1999.

[74] khurana, "Ophthalmology," New Age International - Ophthalmology, vol. 1, pp. 552 pages, 2003.

[75] J.Conrath, "Foveal avascular zone in diabetic retinopathy: quantitativevs qualitative assessment," Eye (Lond), vol. 19, no. 3, pp.322-6, 2004.

[76] R.Bresnick, Condit R, Syrjala S, Palta M, Groo A, and KorthK., "Abnormalities of the fovealavascular zone in diabetic retinopathy,"Arch.Ophthalmol, vol. 102, no. 9, pp. 1286-93, 1994.

[77] G. Richard, "Fluorescein and icg angiography," textbook and atlas,2nd ed. rev. and expanded ed. Georg Thieme Verlag, 1998.

[78] H. A. Nugroho, "Non-invasive image enhancement of colour-retinal fundus image for computerised diabetic retinopathy monitoringand grading system," Phd Thesis Electrical and ElectronicsEngineering Programme, UniversitiTeknologi PETRONAS, vol. 1,pp. 300, 2012.
[79] M.Eladawy, S. M. S. Karawya, M. Elbably, and N. M. A. Salem, "Automatic detection and measurement of foveal avascularzone," Proceedings of the Twentieth National Radio Science Conference, vol. 3, pp. 1-7, 2003.

[80] L Izhar, "Analaysis of retinal vasculature and foveal avascularzone for grading of diabetic retinopathy," M.ScThesis,Electrical and Electronic Engineering UniversitiTeknologiPETRONAS Malaysia, vol. 1, pp. 400, 2006.

[81] R. Guillemaud and M. Brady, "Estimating the bias field of mrimages," IEEE Transactions on Medical Imaging,, vol. 6, no. 3, pp.238-251, 1997.

[82] Ozanian and R. Phillips, "Enhancement of fluoroscopic imageswith varying contrast," Computer Methods and Programs inBiomedicine, vol. 65, no. 1, pp. 1-16, 2001.

[83] Tomazevic, Likar B, and Pernus F, "Comparative evaluation ofretrospective shading correction methods," Journal of Mi-croscopy,vol. 208, no. 3, pp. 212-23, 2002.

[84] A.Salvatelli, G Bizai, G Barbosa, B Drozdowicz, and C Del-rieux, "A comparative analysis of pre-processing techniques in colourretinal images," Journal of Physics: Conference Series, vol. 90, pp.1-7, 2007.

[85] B.Dawant, Zijdenbos AP, and Margolin RA., "Correction ofintensity variations in mr images for computer-aided tissue classification,"IEEE Transactions on Medical Imaging, vol. 12, no. 4,pp. 770-81, 1993.

[86] J.Oakley and B. L. Satherley, "Improving image quality inpoor visibility conditions using a physical model for contrastdegradation," IEEE Transactions on Medical Imaging, vol. 7, no.2, pp. $167-179,1998$.

[87] L. Kubecka, Jiri Jan, and Radim Kolar, "Retrospective illuminationcorrection of retinal images," International Journal ofBiomedical Imaging, vol. 2010, pp. 10 pages, 2010.

[88] J.Sled, Zijdenbos AP, and Evans AC, "A nonparametric methodfor automatic correction of intensity nonuniformity in mri data,"IEEE Transactions on Medical Imaging, vol. 17, no. 1, pp. 87-97,1998.

[89] E. Land and J McCann, "Lightness and retinex theory," Journal ofThe Optical Society of America, vol. 61, no. 1, pp. 1-11, 1971.

[90] R.Gonzalez, "Digital image processing," Upper Saddle RiverNJ:Prentice Hall, 2008.

[91] S. Pizer, John D. Austin, Robert Cromartie, Ari Geselowitz, Bartt. H. Romeny, John B. Zimmerman, and Karel Zuiderveld, "Algorithmsfor adaptive histogram equalization," Physics and En-gineering of Computerized Multidimensional Imaging and ProcessingThomas F. Budinger; Zang-Hee Cho; Orhan Nalcioglu Newport BeachUnited States, vol. 0671, pp. 132, 1986.

[92] E. Pisano, Zong S, Hemminger BM, DeLuca M, Johnston RE,Muller K, Braeuning MP, and Pizer SM., "Contrast limite-dadaptive histogram equalization image processing to im-provethe detection of simulated spiculations in dense mammograms,"Journal of Digital Imaging, vol. 11, no. 4, pp. 193-200, 1998.

[93] Y. Xu, Yansun Xu, J. B. Weaver, D. M. Healy, and Jian $\mathrm{Lu}$, "Wavelet transform domain filters: a spatially selective noisefiltration technique," IEEE Transactions on Image Pro-cessing, vol.3, no. 1057-7149, pp. 6, 1994.

[94] A.Hyvarinen and Oja E, "Independent component analysis:alogrithms and applications," Neural Networks, vol. 13, no. 4,pp. 411-30, 2000.

[95] Ahmad Hani and Toufique Ahmed Soomro, "Non-invasive contrastenhancement for retinal fundus imaging," IEEE InternationalConference on Control System, Computing and Engineering (ICCSCE), vol. 1, pp. 197 - 202, 2013. 
[96] A.Agarwal, "Fundus fluorescein and indocyanine green angi-ography,"DR Annual Report WHO, vol. 16, pp. 168187, 2008.

[97] P.King, K. Hubner, W. Gibbs, and E. Holloway, "Noise identificationand removal in positron imaging systems," , IEEETransactions on Nuclear Science, vol. 28, no. 1, pp. $148-151,1981$

[98] http://www.oculist.net/downaton502/prof/ebook/duanes/ pages/v1/v1c063," .

[99] C. Timothy, "The effects of gain and noise in fundus autofluorescenceimaging," The Journal of Ophthalmic Photography, vol. 28,pp. 111116, 2007.

[100] L. Yannuzzi, Rohrer KT, Tindel LJ, Sobel RS, Costanza MA,Shields W, and Zang E., "Fluorescein angiography complicationsurvey," Arctile on Ophthalmology from UN national Libary ofMedcine, vol. 93, no. 5, pp. 611-7, 1986.

[101] X. Zhou, C. Zhou, and B. G. Stewart, "Comparisons of discretewavelet transform, wavelet packet transform and stationarywavelet transform in denoising pd measurement data," IEEEInternational Symposium on Electrical Insulation, vol. 1, pp. $237-240,2006$.

[102] A. Long, Lepoutre A, Corbillon E, and Branchereau A., "Criticalreview of non- or minimally invasive methods (duplex ultrasonography,mr- and ct-angiography) for evaluating stenosis ofthe proximal internal carotid artery," European journal of vascularand endovascular surgery, vol. 24, no. 1, pp. 43-52, 2002.

[103] M. Folke, Cernerud L, Ekstr?m M, and Hok B., "Critical reviewof non-invasive respiratory monitoring in medical care," Medicaland Biological Engineering and Computing, vol. 41, no. 4, pp. 377-83,2003.

[104] C. Yixin, Delphi Corp, Brighton, and M. Das, "An automatedtechnique for image noise identification using a simple patternclassification approach," 50th Midwest Symposium on Circuits andSystems, vol. 2, pp. $819-822,2007$.

[105] S. Aja-Fernandez, Estepar RS, Alberola-Lopez C, and Westin CF., "Image quality assessment based on local variance," 28th AnnualInternational Conference of the IEEE in Engineering in Medicine andBiology Society,, vol. 1, pp. :4815-8, 2006

[106] Christos M. Michail, Franceschini MA, Zhang Q Ma HY, BallesterosJR, Fantini S, Wallace D, Ntziachristos $\mathrm{V}$, and ChanceB., "Non-invasive and quantitative nearinfrared haemoglobinspectrometry in the piglet brain during hypoxic stress, using afrequency-domain multidistance instrument," Phys. Med. Biol,vol. 46, no. 1, pp. 41-62, 2001.

[107] P. Hansen and Benoit Champagne, "Signal subspace methodsfor speech enhancement," Ph.D Thesis Technical University ofDenmark, vol. 1, pp. 135-159, 1997.

[108] Y. Ephraim and H. L. Van Trees, "A signal subspace approachfor speech enhancement,", IEEE Trans. Speech Audio Process, vol.3, no. 4, pp. 251 - 266, 1995.

[109] Nidal Kamel, Norashikin Yahya, and Aamir S Malik, "Subspacebasedtechnique for speckle noise reduction in sar images," IEEETrans. Geosci. Remote Sens, vol. 13, pp. $154-58,2013$.

[110] D. Brunet and E. Vrscay, "The use of residuals in image denoising,"Image Analysis and Recognition, vol. 1-12, pp. 5627, 2009.

[111] S.Kuo and Johnston JD, "Spatial noise shaping based on humanvisual sensitivity and its application to image coding," IEEETransactions on Image Processing, vol. 11, no. 5, pp. 509-17, 2002.

[112] D.Donoho, "De-noising by soft-thresholding," IEEE Transactionson Information Theory, vol. 41, no. 3, pp. 613 $-627,1995$.
[113] P. Van den Elsen and M. Viergever, "Medical image matchingareview with classification," IEEE Engineering in Medicine andBiology Magazine, vol. 12, no. 1, pp. 26 - 39, 1993.

[114] Heneghan and C Flynn, "Characterization of changes in bloodvessel and tortuosity in retinopathy of prematurity using imageanalysis," Med. Image Anal., vol. 6, no. 4, pp. 407429, 2002.

[115] Chaudhuri, S Chatterje, S.Katz, N. Nelson, and M.Goldbaum, "Detection of retinal blood vessels in retinal images using twodimensionalmatched flters.," IEEE Trans. Med. Imag., vol. 8, no.3, pp. 263 - 269, 1989.

[116] Hoover and A.Kouznetsoz, "Locating blood vessels in retinal imagesby piecewise threshold probing of amatchedflter response,"IEEE Trans. Med. Imag, vol. 19, no. 3, pp. 203-10, 2000.

[117] Lowell, J.Hunter, A. Steel, and D Basu, "Optic nerve headsegmentation," IEEE Transactions on Medical Imaging, vol. 23, no. 2, pp. $256-264,2004$.

[118] Hunter, James Lowell, and Robert E J Ryder, "Non-linear flteringfor vascular segmentation and detection of venous beading,"Technical Report University of Durham, vol. 1, pp. 101-105, 2002.

[119] Tamura, Yasukazu Okamoto, and Kenji Yanashima, "Zerocrossinginterval correction in tracing eye-fundus blood vessels,"PatternRecogn, vol. 21, no. 3, pp. 227233, 1988.

[120] Kochner, Dietrich Schuhmann, Markus Michaelis, and GerdMann, "Course tracking and contour extraction of retinal vesselsfrom colour fundus photographs: most ef?cient use of steerable?lters for model based image analysis.," SPIE 3338, MedicalImaging, vol. 1, pp. 7 pages, 1998.

[121] Teng, M. Lefley, and D. Claremon, "Progress towards automateddiabetic ocular screening: A review of image analysis and intelligentsystems for diabetic retinopathy," Medical and BiologicalEngineering and Computing, vol. 40, no. 1, pp. 2-13, 2002.

[122] Frame, P. E. Undrill, J. A. Olson, and K. C. McHardy, "Structuralanalysis of retinal vessels," Proceedings of the Sixth InternationalConference on Image Processing and its Applications, vol. 2, pp. 824-827, 1996.

[123] Koichiro Akita and Hideki Kuga, "A computer method ofunderstanding ocular fundus images," Pattern Recogn, vol. 5, no.16, pp. 431443, 1982.

[124] Sinthanayothin, C.Boyce, J.F Cook, and H.L.Williamson, "Automatedlocalisation of the optic disc,fovea,and retinal bloodvessels from digital colour fundus images," Br. J. Ophthalmol,vol. 83, no. 8, pp. 902910, 1999.

[125] Heneghan, C.Flynn, J O Keefe, and M.Cahill, "Characterizationof changes in blood vessel and tortuosity in retinopathy ofprematurity using image analysis," Med. Image Anal, vol. 6, pp.407429, 2002.

[126] Gregson, P.H Shen, Z.Scott, and R.C.Kozousek, "Automatedgrading of venous beading," Comput. Biomed. Res, vol. 28, no.4, pp. 291-304, 1995.

[127] Xiaoyi Jiang and D. Mojon, "Adaptive local thresholding byverification-based multithreshold probing with application tovessel detection in retinal images," IEEE Trans Pattern Anal MachIntell, vol. 25, no. 131 - 137, pp. 1, 2003.

[128] Niemeijer, Staal B, and van Ginneken, "Comparative study onretinal vessel segmentation methods on a new publicly availabledatabase.," SPIE Medical Imaging, vol. 5370, pp. 648-656., 2004.

[129] Martinez-Perez, Hughes AD, and Thom SA, "Segmentation ofblood vessels from red-free and fluorescein retinal images," MedImage Anal, vol. 11, no. 1, pp. 47-61, 2007. 
[130] Anzalone, Bizzarri F, Parodi M, and Storace M. A modular,"supervised algorithm for vessel segmentation in red-free retinalimages," Computers in Biology and Medicine, vol. 38, no. 8, pp.913922, 2008.

[131] Espona, Carreira MJ, Penedo MG, and Ortega M, "Retinal vesseltree segmentation using a deformable contour model," 19thInternational Conference on Pattern Recognition (ICPR), vol. 2, pp.1 - 4, 2008.

[132] Cinsdikici and Aydin D, "Detection of blood vessels in ophhalmoscopeimages using mf/ant (matched filter/ant colony)algorithm.," Comput Methods Programs Biomed, vol. 96, no. 2, pp.85-95, 2009.

[133] Vlachos and Dermatas, "Multi-scale retinal vessel segmentationusing line tracking.," Comput Med Imaging Graph, vol. 34, no. 3,pp. 213227, 2009.

[134] Kande, Subbaiah PV, and Savithri TS, "Unsupervised fuzzybased vessel segmentation in pathological digital fundus images,"J Med Syst, vol. 34, no. 5, pp. 849-858, 2009.

[135] Zhang, Zhang L, and Karray, "Retinal vessel extraction bymatched filter with first-order derivative of gaussian," ComputBiol Med, vol. 4, pp. 438-45, 2010.

[136] Xu and Luo S, "A novel method for blood vessel detection fromvretinal images," BioMed Eng Online, vol. 9, no. 1, pp. $1,2010$.

[137] Bankhead, Scholfield CN, McGeown JG, and Curtis TM, "Fastretinal vessel detection and measurement using wavelets andedge location refinement," PLoS ONE, vol. 7, no. 3, pp. 1-12,2012.

[138] Saffarzadeh, Osareh A, and Shadgar, "Vessel segmentation inretinal images using multi-scale line operator and kmeans clustering,"J Med Signals Sens, vol. 4, no. 2, pp. 122-9, 2014.

[139] Chakraborti, Dhiraj K. Jha, Ananda S. Chowdhury, and XiaoyiJiang, "A self-adaptive matched filter for retinal blood vesseldetection. machine vision and applications," Berlin HeidelBerg:Springer Verlag, vol. 26, no. 1, pp. 55-68, 2014.

[140] Bala, M. Ponni, and Vijayachitra, "Extraction of retinal bloodvessels and diagnosis of proliferative diabetic retinopathy usingextreme learning machine," Journal of Medical Imaging and HealthInformatics, Volume 5, Number 2, vol. 5, no. 2, pp. 248-256(9), 2015.

[141] Fujita, T Lee, G N Hayashi, Y Ikedo, Y Gao, and Zhou,"Computer-aided diagnosis: the emerging of three cad systemsinduced by japanese health care needs," Comput. Methods Programs Biomed, vol. 92, no. 3, pp. 238-48., 2008.

[142] Lee, S. C Lee, Kingsley RM,Wang Y, Russell D, Klein $\mathrm{R}$, andWarnA., "Comparison of diagnosis of early retinal lesions of diabeticretinopathy between a computer system and human experts,"Arch. Ophthalmol, vol. 119, no. 4, pp. 509-15, 2001.

[143] Frank and Robert N, "Diabetic retinopathy," Prog. Retin. Eye Res,vol. 350, pp. 48-58, 1995.

[144] Acharya, Eddie YK Ng, and Jasjit Suri, "Image modelling ofhuman eye," Artech House MA, vol. 1, pp. 409, 2008.

[145] Acharya, "Image modeling of human eye," Artech House MA,vol. 1, pp. 409, 2008.

[146] Samuel, Lee ET, Wang Y, Klein R, Kingsley RM, and Warn A.,"Computer classification of a nonproliferative diabetic retinopathy,"Arch. Ophthalmol, vol. 123, no. 6, pp. 759-64., 2005.

[147] Singalavanija, A.Supokavej, J.Bamroongsuk, andP Sinthanayothin, "Feasibility study on computeraidedscreening for diabetic retinopathy," Jounral of Ophthalmol, vol. 50,pp. 361366, 2006.
[148] Kahai, P. Namuduri, K. R, and Thompson H, "A decision supportframework for automated screening of diabetic retinopathy," Int.J. Biomed. Imag, vol. 2, pp. 1630 - 1634, 2006.

[149] Wong, L. Y Acharya, U. R Venkatesh, Y. V Chee, and C Lim., "Identification of different stages of diabetic retinopathy usingretinal optical images," Journal of Information Sciences, vol. 178,no. 1, pp. 106-121, 2008.

[150] Nayak, J.Bhat, and P.S Acharya, "Automated identification ofdifferent stages of diabetic retinopathy using digital fundusimages," Journal of Medical Systems, vol. 32, no. 2, pp. 107-115,2008.

[151] Acharya, Peck Ha Tana, Tavintharan Subramaniam, ToshiyoTamura, Kuang Chua Chua, SeachChyr Ernest Goh, Choo MinLim, Shu Yi Diana Goh, and Kang Rui Conrad Chung, "Automatedidentification of diabetic type 2 subjects with and withoutneuropathy using wavelet transform on pe-dobarograph," J. Med.Syst., vol. 32, no. 1, pp. 21-29, 2008.

[152] Nicolai, L Jannik, G. Michael, G.Henrik, and Michael, "Automateddetection of diabetic retinopathy in a fundus photographicscreening population," Invest. Ophthalmol. Vis. Sci, vol. 44, no. 2,pp. 767-71, 2003.

[153] Neubauer and Chryssafis, "Screening for diabetic retinopathyand optic disc topography with the retinal thickness analyzer,"Ophthalmologe, vol. 102, no. 3, pp. 251-8, 2005.

[154] Lee, Wang, Klein R, Kingsley RM, and Warn A, "Computerclassification of nonproliferative diabetic retinopathy," Arch.Ophthalmol, vol. 123, no. 6, pp. 759-64, 2005.

[155] Estabridis and de Figueiredo, "Automatic detection and diagnosisof diabetic retinopathy," IEEE Int. Conf. Image Processing ICIP,vol. 2, pp. 445 - 448, 2007.

[156] Li and Jin, "Screening diabetic retinopathy through color retinalimages.," Proceedings of the 1st international conference on Medicalbiometrics, vol. 1, pp. 176-183, 2008.

[157] Abrmoff, Niemeijer M, Suttorp-Schulten MS, Viergever MA,Russell SR, and van Ginneken B., "Evaluation of a system forautomatic detection of diabetic retinopathy from color fundusphotographs in a large population of patients with diabetes,"Diabetes Care, vol. 31, no. 2, pp. 193-8, 2008.

[158] Vujosevic, Cavarzeran, F Avogaro, and Midena, "Screeningfor diabetic retinopathy: 1 and 3 nonmydriatic 45-degree digitalfundus photographs vs 7 standard early treatment diabeticretinopathy study fields," Am. J. Ophthalmol., vol. 148, no. 1, pp.111-8, 2009.

[159] Enrique Soto-Pedre, Amparo Navea, Saray Millan, Maria C.Hernaez-Ortega, JesusMorales, Maria C Desco, and Pablo, "Evaluation of automated image analysis software for the detection ofdiabetic retinopathy to reduce the ophthalmologists workload,"ActaOphthalmologica, vol. 93, no. 1, pp. 52-56, 2015. 